\title{
Caracterización de la generación del milenio en el contexto laboral: una revisión de la literatura
}

\author{
Lina María Ramírez Angel*
}

Fecha de recibido: 09 de septiembre de 2019

Fecha de aprobado: 26 de octubre de 2021

Para citar este artículo: Ramírez Angel, L. M. (2022). Caracterización de la generación del milenio en el contexto laboral: una revisión de la literatura. Revista Universidad E Empresa, 24(42), 1-37. https://doi.org/10.12804/revistas.urosario.edu.co/

empresa/a.8230

\section{Resumen}

Actualmente, tres generaciones de trabajadores están presentes en las organizaciones; la generación de los baby boomers, está a punto de salir del mercado laboral y le abren paso a la generación de trabajadores millennials. El objetivo de este artículo es presentar una revisión sistemática de la literatura referente a la generación del milenio, destacando: primero, la caracterización de los millennials, que contiene elementos clasificados en psicológicos, familiares y sociales que han demostrado tener efecto en el contexto laboral; y segundo, la extracción de prácticas que han dado algunos autores para retenerlos y evitar altos costos por la pérdida de productividad, contratación y formación de nuevos empleados. De esta revisión se concluye que los millennials son aquellas personas nacidas entre 1980 y el 2000, quienes en el contexto laboral buscan tener equilibrio entre su vida y el trabajo, retroalimentación, contacto con los líderes y crecimiento rápido a posiciones altas. Además, prefieren laborar en y para organizaciones que les generen aprendizaje y los desafíen, razón por la que cambian constantemente de trabajo. En consecuencia, es importante entender que en las prácticas de retención no es necesario realizar grandes inversiones de dinero, sino mejorar y fortalecer los procesos del área de recursos humanos.

Palabras clave: Millennials; Generación Y; características de la Generación Y; retención de empleados.

\footnotetext{
Magister en Administración y estudiante del doctorado en Administración de la Universidad Nacional de Colombia, sede Bogotá, integrante del Grupo Salud-Trabajo y del Grupo Interdisciplinario en Teoría e Investigación Aplicada en Ciencias Económicas (GITIACE). Docente ocasional Universidad Nacional de Colombia, Bogotá. Correo electrónico: limramirezan@ unal.edu.co ORCID: https://orcid.org/0000-0003-1253-9292
} 


\title{
Characterization of Millennials in the Labor Context: A Literature Review
}

\begin{abstract}
Currently, three generations of employees work together in organizations: baby boomers are about to leave the labor market and make way for millennials. This article presents a systematic literature review that highlights two aspects: the characterization of millennial workers, which contains psychological, family, and social elements that have shown to have an effect in the work context, and the extraction of retention practices that different authors have proposed to avoid high costs due to loss productivity and hiring and training new employees. From this review, it is concluded that millennials are those born between 1980 and 2000, who, in the labor context, seek a balance between life and work, feedback, contact with leaders, and rapid growth to high positions. It can also be concluded that millennials prefer jobs that generate learning and are challenging, due to which they constantly change jobs. In response to that, it is important that companies understand that in retention practices, it is not necessary to make large money investments but to improve and strengthen the processes around human resources.
\end{abstract}

Keywords: Millennials; Generation Y; Generation Y characteristics; employee retention.

\section{Caracterização da geração do milênio no contexto laboral: uma revisão da literatura}

\section{Resumo}

Atualmente, três gerações de trabalhadores estão presentes nas organizações; a geração dos baby boomers está prestes a deixar o mercado de trabalho e abrir caminho para a geração de trabalhadores millennials. O objetivo deste artigo é apresentar uma revisão sistemática da literatura referente à geração millennial, destacando: primeiro, a caracterização dos millennials que contém elementos classificados como psicológicos, familiares e sociais que demonstraram ter efeito no contexto laboral e, segundo, a extração de práticas que alguns autores têm dado para retê-los e evitar altos custos devido à perda de produtividade, contratação e treinamento de novos funcionários. A partir dessa revisão, conclui-se que os millennials são aquelas pessoas nascidas entre 1980 e 2000 , as quais no contexto laboral buscam ter equilíbrio entre vida pessoal e profissional, retroalimentação, contato com lideranças e rápida ascenção para altos cargos. Além disso, preferem trabalhar em e para organizações que geram aprendizado e os desafiam; razão pela qual mudam constantemente de emprego. Consequentemente, é importante entender que nas práticas de retenção não é necessário fazer grandes investimentos de dinheiro, mas sim melhorar e fortalecer os processos da área de recursos humanos.

Palavras-chave: Millennials, Geração Y; características de geração; retenção de funcionários.

\section{Introducción}

Para el 2018, más del 32\% de la población tenía un rango de edad entre 20 a 39 años según el tercer comunicado de prensa del último censo nacional de población y vivienda (CNPv) (DANE, 2019) y según el estimado de proyección de la misma entidad, para el 2020 se llegó a cerca de 15864936 personas que estarían en el mismo rango de edad. Actualmente, el personal de las organizaciones está conformado por tres generaciones de trabajadores: los 
baby boomers, la Generación X y la Generación Y (Millennials). La generación de los baby boomers está cerrando su ciclo productivo, es decir, está saliendo del mercado laboral, pues los de menor edad en esta generación tienen 57 años, por lo que en poco tiempo alcanzarán su edad de pensión. Este panorama evidencia que el relevo generacional será inminente. Es necesario entender que en las empresas interactúan diversas generaciones, cada una con distintas expectativas, que buscan satisfacción laboral y desarrollo de carrera de formas diferentes. El reto para las organizaciones será aprender a gestionar a los trabajadores de la Generación Y, ya que ellos serán quienes cubran las vacantes y según la literatura, tienden a rotar de puesto de trabajo (Plessis et al., 2015), entre otras cosas para aprender constantemente (Lyons et al., 2012; Thompson \& Gregory, 2012).

Según la Gran encuesta integrada de hogares, solo en Bogotá cerca del 43\% de los trabajadores que en ese momento tenían entre 17 a 37 años, tenían menos de 1 año en el cargo que estaban ocupando en ese momento y el $47 \%$ del mismo segmento habían permanecido tan solo 1 año o menos en su trabajo anterior (DANE, 2017). En la literatura se menciona que estos trabajadores están tan impacientes por avanzar que no esperan más de dos años para ser promovidos (Pasko et al., 2021). La constante rotación de trabajadores incrementa los costos para las organizaciones, costos asociados a procesos de selección, capacitación, pérdida de productividad, sobrecarga laboral a otros trabajadores, pérdida de talento y de conocimiento, degradación en el servicio y ruptura del flujo con los clientes (Hassan et al., 2021; Kuyken, 2012; Pasko et al., 2021). Se estima que esos costos son más del 150 por ciento del salario del trabajador y es por esto que se considera esencial generar estrategias de gestión innovadoras para retener a la Generación Y (Hassan et al., 2020).

Si bien el presente estudio no se centró en la coyuntura actual del Covid-19, es necesario destacar que en la literatura se han mencionado algunas modificaciones en cuanto a la rotación de trabajadores millennials. Ante la cuarentena y el aislamiento preventivo, se presentaron cierres generalizados que afectaron la economía a nivel mundial, siendo este un argumento para que quienes pensaban en cambiar de trabajo lo reconsideraran y postergaran la decisión. Pero, por otro lado, quienes presentaron altos niveles de estrés, agotamiento, inestabilidad en cuanto a salud mental, sobrecarga de trabajo, etc., consideraron la intensión de rotar y en algunos casos optaron por cambiar de empleo (Prihadi et al., 2021). 
Teniendo en cuenta lo anterior, el objetivo de este artículo es presentar, a partir de una revisión de literatura, cuáles son las características de la generación del milenio, diferenciando cuáles se han contrastado de manera empírica y cuáles de manera teórica. A partir de ello, mostrar cómo sus características psicológicas, familiares y sociales han influido en su comportamiento en el ámbito laboral y, finalmente, recopilar de diversos autores estrategias para la retención de estos trabajadores.

Este escrito se estructura a partir de cuatro apartados: el primero presenta los hallazgos en cuanto al origen y definición del término millennial; el segundo corresponde a la estrategia de búsqueda y revisión sistemática de la literatura; el tercero presenta una caracterización de la Generación Y (en el ámbito social, familiar, psicológico y laboral) según algunos autores; $y$, finalmente, se sintetizan las recomendaciones de los autores para generar estrategias de retención de millennials.

\section{Origen y términos asociados a los Millennials}

La teoría generacional nace a partir de tres autores: por un lado, Mannheim (1952) en Alemania, con "El problema de las Generaciones"; y por otro, Strauss y Howe (1991) con su libro "Generaciones" en Estados Unidos, en el que toman la historia de la nación norteamericana como una serie de relevos generacionales. En este sentido, el primer autor define las generaciones por su situación en el curso de la historia, pues "Un grupo generacional comparte experiencias de vida históricas y sociales, que afectan la forma en que las personas de esa generación desarrollan y distinguen un grupo generacional de otro" (Smola \& Sutton, 2002, p. 364); al compartir ciertos valores, historia, lenguaje, creencias y normas, las cohortes generacionales pueden considerarse como un tipo de grupo cultural (Glazer et al., 2019).

La cohorte generacional millennial, también denominada Generación Y, toma como rasgo común el rango de años en que nacieron. Según los autores de la revisión de literatura que se presentará a lo largo de este documento, el periodo inicia entre 1977 y 1983 y culmina entre 1992 y 2005. Teniendo en cuenta que no existe un consenso sobre las 
fechas exactas que esta generación comprende, para efectos de este artículo, se determina un promedio redondeado hacia arriba y se toman las fechas de 1980 al 2000 (anexo 1).

Esta generación recibe nombres tales como Millennials (por haber vivido su niñez al inicio del milenio); Generation Y (por la continuidad con la generación anterior la X); Nexter o the Nexus Generation, Baby Boom Echo (por tener características en común con los Baby boomers) (Glass, 2007; Ng et al., 2010); GenMe, (refiriéndose a su egocentrismo) (Barkhuizen, 2014; Twenge, 2010; Twenge \& Campbell, 2008); y Generación WHY, (por la analogía fonética con la letra Y en Inglés y como expresión del carácter crítico que suele caracterizar a la mayoría de la generación) (García Lombardía et al., 2008). Otras denominaciones incluyen Generación de Internet o e-generation; My Pod; generación now; generación despierta (De \& Su, 2011); Wannabees; la Generación-Feel Good y Cyberkids (Stanimir, 2015). Si bien esta generación es denominada de muchas maneras que se agrupan en la figura 1, las más aceptadas y popularizadas son Millennials y Generación Y. Por tanto, estas dos últimas denominaciones se utilizarán a lo largo de este documento.

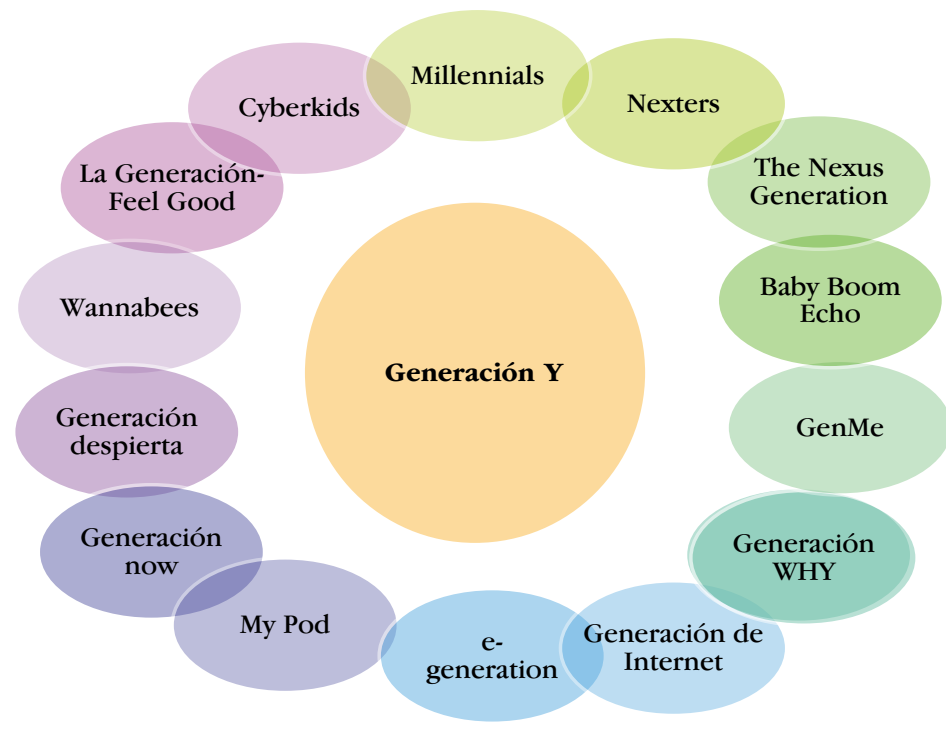

Figura 1. Nombres asociados a la Generación Y

Fuente: elaboración propia a partir de la información extraida de Glass (2007); Ng et al. (2010); Barkhuizen (2014);

Twenge (2010); Twenge y Campbell (2008), García Lombardía et al. (2008); De y Su (2011); y Stanimir (2015). 


\section{Metodología}

\subsection{Revisión sistemática de literatura}

La revisión sistemática de la literatura (RSL) es adoptada para este estudio debido al enfoque riguroso que adopta. Este tipo de revisión a menudo se utiliza para responder preguntas de investigación altamente estructuradas y específicas, "tiene como objetivo identificar toda la literatura en el área temática para garantizar que no se omitan conocimientos existentes" (Arshed \& Danson, 2015, p. 32). La RsL es un método que examina de manera cuidadosa y efectiva la investigación y conocimiento ya existente sobre un tema en específico; el objetivo es lograr una síntesis de los hallazgos, a partir de un gran número de estudios sobre un problema en particular.

El método puede ser replicable; por esto, es necesario tener claridad en la estrategia de investigación y los criterios de inclusión y exclusión (Ridley, 2012). En otras palabras, es un proceso que implica organización, clasificación, análisis y síntesis, que permite dominar con rigurosidad un tema específico. La revisión sistemática de literatura (RSL) se construye a través de estudios publicados y seleccionados con métodos replicables, lo que hace que el proceso sea confiable (Chicaíza-Becerra et al., 2017).

\subsection{Etapas del proceso metodológico}

La Revisión Sistemática de Literatura se lleva a cabo en tres grandes etapas. En la primera, se elabora el protocolo que guía el proceso de revisión. Esta etapa le permite al investigador conocer a fondo un tema, sus antecedentes y categorías y, así mismo, le brinda herramientas para establecer su pregunta de investigación. La segunda etapa es la revisión en sí misma, que necesariamente debe tener una estrategia de búsqueda clara, como la selección de palabras clave, la ecuación de búsqueda, las bases de datos y los criterios de inclusión y exclusión para seleccionar los documentos. Finalmente, la tercera se refiere a la creación y divulgación del reporte o resultado de la investigación (Chicaíza-Becerra et al., 2017). 


\subsection{Estrategia de búsqueda y revisión sistemática de la literatura}

Para dar inicio al proceso de investigación, se realizó una búsqueda de tesauros relacionados con todos los términos asociados a la generación del milenio. De igual manera, con los términos empleo, retención, trabajador y trabajo. Se realizaron diferentes combinaciones de ecuaciones de búsqueda descartando tesauros que no fueran relevantes e incluyendo palabras clave como millennials, generation y, employee retention, job y employment.

La búsqueda se generó primero en Scopus, una base de datos muy completa que abarca varias disciplinas y cuenta con una gran variedad de revistas y con artículos no solo recientes sino también antiguos. Para complementar la búsqueda, se seleccionaron dos bases de datos bibliográficas adicionales: Web of Science (con una mayor cobertura en años) y Ebsco (con amplia cobertura en textos completos). Para tener un mayor control del proceso de investigación, la tabla 1 evidencia la fecha de búsqueda y el número de artículos que arrojó la ecuación de búsqueda.

Tabla 1. Resultados de la búsqueda en bases de datos bibliográficas

\begin{tabular}{|c|c|c|c|c|c|}
\hline \# & $\begin{array}{c}\text { Base de } \\
\text { datos }\end{array}$ & $\begin{array}{c}\text { Fecha de } \\
\text { actualización }\end{array}$ & Ecuación & Limitante/filtro & $\begin{array}{c}\text { Resultados 09- } \\
2021\end{array}$ \\
\hline 1 & Scopus & 29/09/2021 & $\begin{array}{l}\text { (Millennials OR "generation } \\
\text { y") AND ("employee reten- } \\
\text { tion" OR job* OR employ- } \\
\text { ment) }\end{array}$ & $\begin{array}{l}\text { Search Field Type } \\
\text { Article title, Abstract, } \\
\text { Keywords }\end{array}$ & 526 \\
\hline 2 & $\begin{array}{l}\text { Web of } \\
\text { science }\end{array}$ & 29/09/2021 & 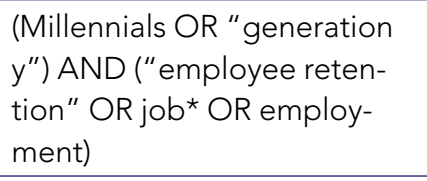 & Tema & 290 \\
\hline \multirow[t]{2}{*}{3} & Ebsco & 29/09/2021 & $\begin{array}{l}\text { (Millennials OR "generation } \\
\left.\mathrm{y}^{\prime \prime}\right) \text { AND (employee retention } \\
\text { OR job* OR employment) }\end{array}$ & $\begin{array}{l}\text { 1) Publicaciones académicas } \\
\text { 2) Idioma: Inglés } \\
\text { Español }\end{array}$ & 165 \\
\hline & & & & TOTAL & 981 \\
\hline
\end{tabular}

Fuente: elaboración propia con base en el número de artículos de las bases de Scopus, Wos y Ebsco. 


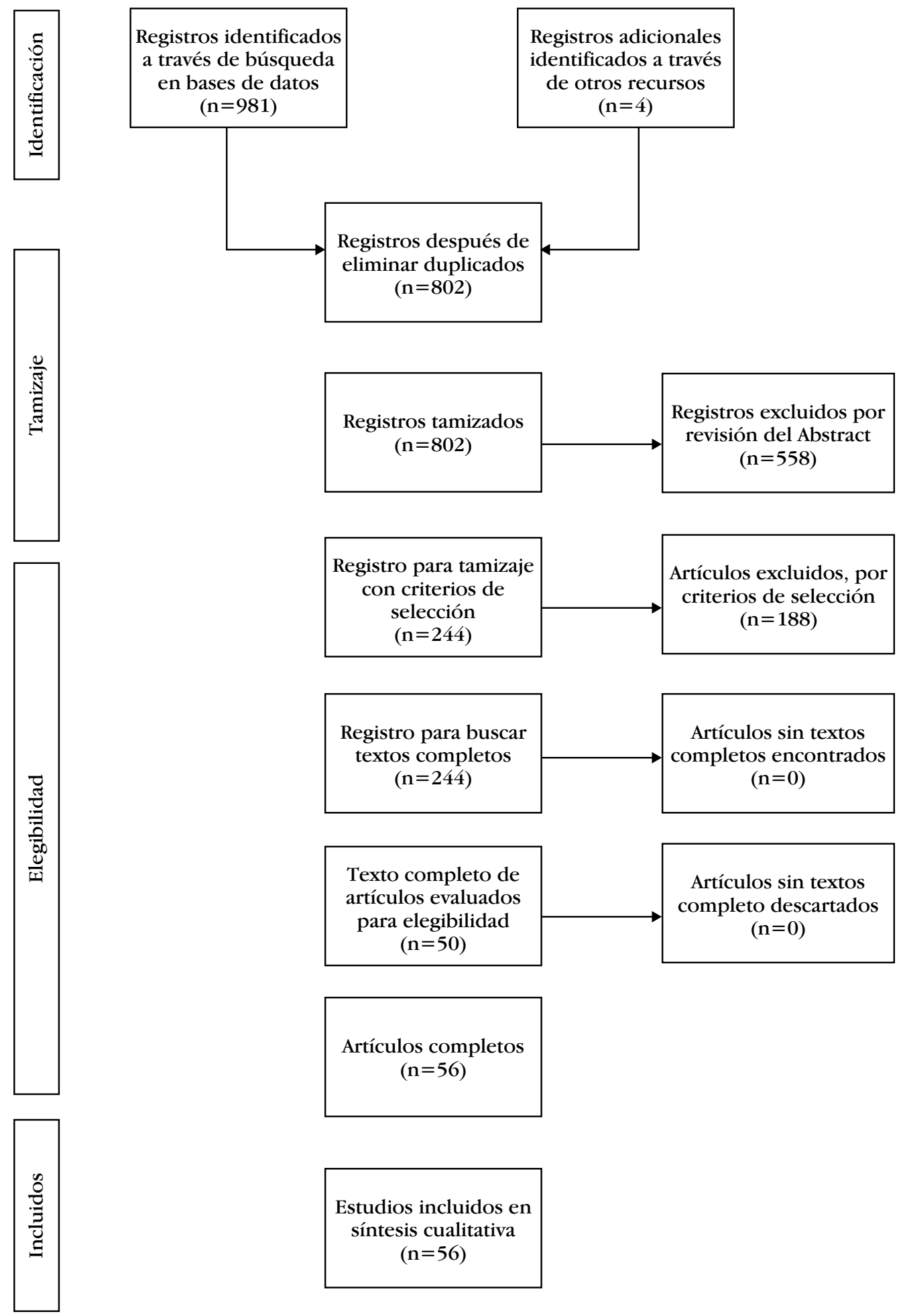

Figura 2. Diagrama de flujo Prisma, estrategia de búsqueda

Fuente: elaboración propia a partir de la revisión de literatura. 
Gracias a la primera revisión de literatura, tesauros y un barrido completo de los resúmenes de los 806 artículos únicos, se establecieron los criterios de inclusión y exclusión para la investigación y la forma como se podrían clasificar para facilitar su lectura.

\subsection{Criterios de inclusión}

Se dio prioridad a los artículos referentes a la definición de la generación del milenio, origen del concepto, rango de años de nacimiento de la Generación Y, y características asociadas a la misma cohorte generacional. De igual forma, se identificó como prioritario todo lo relacionado con la retención de trabajadores, incentivos, desarrollo de carrera y satisfacción laboral.

\subsection{Criterios de exclusión}

Se dejaron de lado términos que hacían referencia a otras investigaciones, como educación y marketing, y enfoques puntuales a sectores en específico, como el turístico y la salud. También se excluyeron libros completos y conferencias.

\subsection{Clasificación de los artículos}

Luego de reducir los artículos según los criterios de inclusión y exclusión, se procedió a clasificarlos de acuerdo con características, comparaciones entre generaciones, retención de trabajadores millennials y casos puntuales en sectores (se resaltan aquellos que tienen que ver con el sector salud y turismo solo para saber y dimensionar la cantidad de artículos relacionados).

A medida que se realizaba la lectura completa de los artículos seleccionados, se elaboró una matriz de extracción con los elementos destacables de cada artículo y se logró clasificar las características de la Generación Y en cuatro grupos: características psicológicas, familiares, sociales y de comportamiento en el ámbito laboral. Adicional a esto, se buscó identificar las mejores prácticas de retención según los autores, para luego organizarlas y seleccionarlas para este artículo (se asoció cada práctica de retención a una o más características de la nueva generación de trabajadores, tal como se verá más adelante en el apartado de resultados). 


\section{Resultados}

Se encontró que cerca del $59 \%$ de la bibliografía obtenida son trabajos empíricos, 34\% teóricos y $7 \%$ teórico-empíricos. Veintisiete de los artículos analizados trabajan con encuestas y tan solo seis realizan entrevistas; tres son análisis de datos ya existentes y dos contienen grupos focales dentro de las investigaciones. Los sectores más estudiados hasta el momento, en relación con esta generación, son el sector salud y el sector del turismo. El primero porque, según se menciona en los artículos, ha disminuido el número de trabajadores en el campo de la salud (médicos generales o de diferentes especialidades y enfermeras), y se buscan diferentes prácticas para retenerlos. Por otro lado, se ha investigado el sector turístico debido a la alta demanda de empleo, que, en la actualidad, se presenta en este sector.

A continuación, la tabla 2 presenta un resumen de los autores recopilados mediante la revisión sistemática de literatura. Allí se identifica si el estudio es teórico o empírico, el año de publicación, si introduce el término de millennial, si menciona características psicológicas, familiares, sociales o laborales y si indican alguna práctica para la retención de esta generación en el trabajo.

Tabla 2. Resumen de autores presentes en la revisión sistemática de literatura

\begin{tabular}{|c|c|c|c|c|c|c|c|c|c|c|c|c|}
\hline \multirow[b]{3}{*}{ \# } & \multirow[b]{3}{*}{ Base } & \multirow[b]{3}{*}{ Autor } & \multirow[b]{3}{*}{ Título } & \multirow[b]{3}{*}{ Año } & \multirow[b]{3}{*}{$\begin{array}{l}\text { Lugar del } \\
\text { estudio }\end{array}$} & \multirow[b]{3}{*}{$\begin{array}{l}\text { Tipo de } \\
\text { Estudio }\end{array}$} & \multirow[b]{3}{*}{ 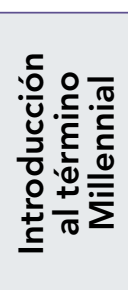 } & \multirow{2}{*}{\multicolumn{4}{|c|}{ Características }} & \\
\hline & & & & & & & & & & & & \multirow[b]{2}{*}{ 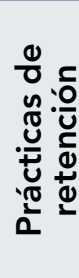 } \\
\hline & & & & & & & & $\begin{array}{l}\frac{n}{70} \\
\frac{u}{\delta} \\
\frac{0}{0} \\
\cdot \frac{u}{n} \\
0\end{array}$ & 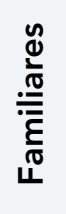 & $\begin{array}{l}\frac{\mathscr{d}}{\pi} \\
\frac{\pi}{U} \\
\dot{0}\end{array}$ & 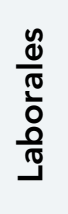 & \\
\hline 1 & Ebsco & Navós, Oscar & $\begin{array}{l}\text { Nuevas generaciones en las } \\
\text { empresas: algunas claves } \\
\text { para su gestión. }\end{array}$ & 2015 & Chile & Teórico & $x$ & & $x$ & $x$ & $x$ & $x$ \\
\hline 2 & Wos & $\begin{array}{l}\text { Ng, ESW; } \\
\text { Schweitzer, L; } \\
\text { Lyons, ST }\end{array}$ & $\begin{array}{l}\text { New Generation, Great } \\
\text { Expectations: A Field Study } \\
\text { of the Millennial Generation }\end{array}$ & 2010 & Canadá & Empírico & $x$ & $x$ & & $x$ & $x$ & \\
\hline 3 & Scopus & $\begin{array}{l}\text { Howe N., } \\
\text { Strauss W. }\end{array}$ & $\begin{array}{l}\text { The next } 20 \text { years: How } \\
\text { customer and workforce } \\
\text { attitudes will evolve }\end{array}$ & 2007 & EEUU & Teórico & $x$ & & $x$ & $x$ & $x$ & \\
\hline
\end{tabular}




\begin{tabular}{|c|c|c|c|c|c|c|c|c|c|c|c|c|}
\hline & & & & & & & & & & & & \\
\hline & & & & & & & & Car & racte & eríst & cas & \\
\hline \# & Base & Autor & Título & Año & $\begin{array}{l}\text { Lugar del } \\
\text { estudio }\end{array}$ & $\begin{array}{l}\text { Tipo de } \\
\text { Estudio }\end{array}$ & 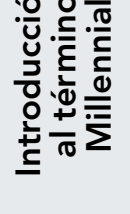 & $\begin{array}{l}\frac{n}{0} \\
\frac{0}{0} \\
\frac{0}{0} \\
\frac{u}{n} \\
0\end{array}$ & 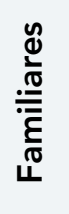 & 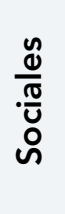 & $\begin{array}{l}\frac{\mathscr{0}}{\pi} \\
\frac{0}{0} \\
\frac{0}{\pi}\end{array}$ & 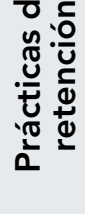 \\
\hline 4 & Scopus & $\begin{array}{l}\text { Twenge J.M., } \\
\text { Campbell } \\
\text { S.M. }\end{array}$ & $\begin{array}{l}\text { Generational differences } \\
\text { in psychological traits and } \\
\text { their impact on the work- } \\
\text { place }\end{array}$ & 2008 & EEUU & Teórico & $x$ & $x$ & $x$ & & $x$ & $x$ \\
\hline 5 & Ebsco & $\begin{array}{l}\text { Pilar García } \\
\text { Lombardía, } \\
\text { Guido } \\
\text { Stein, José } \\
\text { Ramón Pin }\end{array}$ & $\begin{array}{l}\text { Motivaciones y valores de la } \\
\text { Generación Y. }\end{array}$ & 2008 & España & Teórico & $x$ & & & $x$ & & $x$ \\
\hline 6 & Scopus & De H., Su X. & $\begin{array}{l}\text { Managing generation Y: } \\
\text { Recruiting and motivating }\end{array}$ & 2011 & China & Teórico & $x$ & & $x$ & $x$ & & $x$ \\
\hline 7 & Scopus & $\begin{array}{l}\text { Thompson C., } \\
\text { Gregory J.B. }\end{array}$ & $\begin{array}{l}\text { Managing Millennials: A } \\
\text { Framework for Improving } \\
\text { Attraction, Motivation, and } \\
\text { Retention }\end{array}$ & 2012 & Inglaterra & Teórico & & $x$ & $x$ & $x$ & $x$ & $x$ \\
\hline 8 & Scopus & $\begin{array}{l}\text { Jonck P., van } \\
\text { der Walt F., } \\
\text { Sobayeni N.C. }\end{array}$ & $\begin{array}{l}\text { A generational perspective } \\
\text { on work values in a South } \\
\text { African sample }\end{array}$ & 2017 & Sudáfrica & Empírico & & $x$ & & $x$ & $x$ & \\
\hline 9 & Scopus & Stanimir A. & $\begin{array}{l}\text { Generation } Y \text { - character- } \\
\text { istics of attitudes on labour } \\
\text { market }\end{array}$ & 2015 & Polonia & Teórico & $x$ & & & & $x$ & \\
\hline 10 & Scopus & Barkhuizen N. & $\begin{array}{l}\text { Exploring the importance of } \\
\text { rewards as a talent manage- } \\
\text { ment tool for Generation Y } \\
\text { employees }\end{array}$ & 2014 & Sudáfrica & Empírico & $x$ & $x$ & & & $x$ & $x$ \\
\hline 11 & Wos & Twenge, JM & $\begin{array}{l}\text { A Review of the Empirical } \\
\text { Evidence on Generational } \\
\text { Differences in Work } \\
\text { Attitudes }\end{array}$ & 2010 & $\begin{array}{l}\text { Abarca } \\
\text { a EEUU, } \\
\text { Australia } \\
\text { y algunos } \\
\text { países de } \\
\text { Europa }\end{array}$ & Empírico & $x$ & & & $x$ & & \\
\hline 12 & Ebsco & $\begin{array}{l}\text { Pilar García } \\
\text { Lombardía, } \\
\text { Guido } \\
\text { Stein, José } \\
\text { Ramón Pin }\end{array}$ & $\begin{array}{l}\text { ¿A qué generación } \\
\text { pertenece usted? }\end{array}$ & 2011 & Uruguay & Teórico & & $x$ & & & & $x$ \\
\hline 13 & Ebsco & $\begin{array}{l}\text { Federico } \\
\text { Muttoni }\end{array}$ & $\begin{array}{l}\text { Buscan empresas donde } \\
\text { sentirse bien y desarrollarse. }\end{array}$ & 2011 & Uruguay & Teórico & & $x$ & & & $x$ & \\
\hline 14 & Ebsco & Rayan, L. & Generación Y, en el día a día. & 2011 & Uruguay & Teórico & & $x$ & & & $x$ & $x$ \\
\hline 15 & Scopus & Glass A. & $\begin{array}{l}\text { Understanding generational } \\
\text { differences for competitive } \\
\text { success }\end{array}$ & 2007 & EEUU & Teórico & $x$ & & $x$ & $x$ & & \\
\hline
\end{tabular}




\begin{tabular}{|c|c|c|c|c|c|c|c|c|c|c|c|c|}
\hline & & & & & & & \multirow{2}{*}{\multicolumn{5}{|c|}{ Características }} & \multirow[b]{3}{*}{ 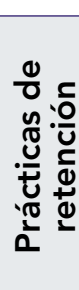 } \\
\hline \multirow[b]{2}{*}{ \# } & \multirow[b]{2}{*}{ Base } & \multirow[b]{2}{*}{ Autor } & \multirow[b]{2}{*}{ Título } & \multirow[b]{2}{*}{ Año } & \multirow[b]{2}{*}{$\begin{array}{l}\text { Lugar del } \\
\text { estudio }\end{array}$} & \multirow[b]{2}{*}{$\begin{array}{l}\text { Tipo de } \\
\text { Estudio }\end{array}$} & & & & & & \\
\hline & & & & & & & 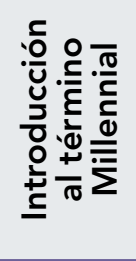 & $\begin{array}{l}\frac{n}{0} \\
\frac{0}{0} \\
\frac{0}{0} \\
\frac{u}{n} \\
\frac{0}{0}\end{array}$ & 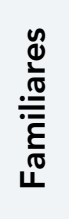 & $\begin{array}{l}\frac{\mathscr{d}}{\pi} \\
\frac{\pi}{4} \\
\dot{0}\end{array}$ & $\begin{array}{l}\frac{\mathscr{d}}{\pi} \\
\frac{0}{0} \\
\frac{0}{0}\end{array}$ & \\
\hline 16 & Scopus & $\begin{array}{l}\text { Plessis L.D., } \\
\text { Barkhuizen } \\
\text { N., Stanz K., } \\
\text { Schutte N. }\end{array}$ & $\begin{array}{l}\text { The management side of } \\
\text { talent: Causal implications } \\
\text { for the retention of genera- } \\
\text { tion y employees }\end{array}$ & 2015 & Sudáfrica & Empírico & & & & & & $x$ \\
\hline 17 & Scopus & $\begin{array}{l}\text { Bissola R., } \\
\text { Imperatori B. }\end{array}$ & $\begin{array}{l}\text { Generation Y at work: The } \\
\text { role of e-HRM in building } \\
\text { positive work attitudes }\end{array}$ & 2010 & Italia & Empírico & & & & & & $x$ \\
\hline 18 & Scopus & $\begin{array}{l}\text { Kowske B.J., } \\
\text { Rasch R., } \\
\text { Wiley J. }\end{array}$ & $\begin{array}{l}\text { Millennials' (lack of) attitude } \\
\text { problem: An empirical } \\
\text { examination of generational } \\
\text { effects on work attitudes }\end{array}$ & 2010 & EEUU & Empírico & & & & & $x$ & \\
\hline 19 & Scopus & $\begin{array}{l}\text { Lyons S.T., } \\
\text { Schweitzer } \\
\text { L., Ng E.S.W., } \\
\text { Kuron L.K.J. }\end{array}$ & $\begin{array}{l}\text { Comparing apples to apples: } \\
\text { A qualitative investigation } \\
\text { of career mobility patterns } \\
\text { across four generations }\end{array}$ & 2012 & Canadá & Empírico & & & & & $x$ & \\
\hline 20 & Scopus & $\begin{array}{l}\text { Kim H., } \\
\text { Knight D.K., } \\
\text { Crutsinger C. }\end{array}$ & $\begin{array}{l}\text { Generation Y employees' } \\
\text { retail work experience: The } \\
\text { mediating effect of job } \\
\text { characteristics }\end{array}$ & 2009 & EEUU & Empírico & & & & & & $x$ \\
\hline 21 & Scopus & Kuyken K. & $\begin{array}{l}\text { Knowledge communities: } \\
\text { Towards a re-thinking of in- } \\
\text { tergenerational knowledge } \\
\text { transfer }\end{array}$ & 2012 & Canadá & Teórico & & & & & & $x$ \\
\hline 22 & Scopus & $\begin{array}{l}\text { Carpenter } \\
\text { M.J., de } \\
\text { Charon L.C. }\end{array}$ & $\begin{array}{l}\text { Mitigating multigeneration- } \\
\text { al conflict and attracting, } \\
\text { motivating, and retaining } \\
\text { millennial employees by } \\
\text { changing the organizational } \\
\text { culture: A theoretical model }\end{array}$ & 2014 & EEUU & Empírico & & & & & & $x$ \\
\hline 23 & Wos & Levenson A.R. & $\begin{array}{l}\text { Millennials and the World } \\
\text { of Work: An Economist's } \\
\text { Perspective }\end{array}$ & 2010 & EEUU & Teórico & & & & & $x$ & \\
\hline 24 & Wos & $\begin{array}{l}\text { Kleinhans } \\
\text { K.A., } \\
\text { Chakradhar } \\
\text { K., Muller S., } \\
\text { Waddill P. }\end{array}$ & $\begin{array}{l}\text { Multigenerational percep- } \\
\text { tions of the academic work } \\
\text { environment in higher edu- } \\
\text { cation in the United States }\end{array}$ & 2015 & EEUU & Empírico & & & & & & $x$ \\
\hline 25 & Ebsco & Gallicano T.D. & $\begin{array}{l}\text { Relationship management } \\
\text { with the Millennial gener- } \\
\text { ation of public relations } \\
\text { agency employees. }\end{array}$ & 2013 & EEUU & Empírico & & & & & & $x$ \\
\hline
\end{tabular}




\begin{tabular}{|c|c|c|c|c|c|c|c|c|c|c|c|c|}
\hline & \multirow{2}{*}{\multicolumn{5}{|c|}{ Características }} & \multirow[b]{3}{*}{ 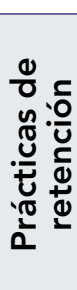 } \\
\hline & & & & & & & & & & & & \\
\hline \# & Base & Autor & Título & Año & $\begin{array}{l}\text { Lugar del } \\
\text { estudio }\end{array}$ & $\begin{array}{l}\text { Tipo de } \\
\text { Estudio }\end{array}$ & 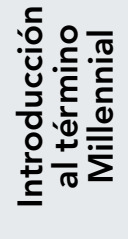 & \multicolumn{2}{|c|}{ 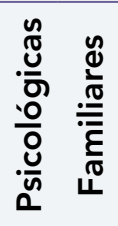 } & $\begin{array}{l}\frac{\mathscr{d}}{\pi} \\
\frac{\pi}{U} \\
\stackrel{\circ}{0}\end{array}$ & $\begin{array}{l}\frac{\mathscr{d}}{\pi} \\
\frac{0}{0} \\
\frac{0}{0}\end{array}$ & \\
\hline 26 & Ebsco & $\begin{array}{l}\text { Bell, Nancy } \\
\text { Sutton; } \\
\text { Connell, J. } \\
\text { A.; McMinn, } \\
\text { Nathan E. }\end{array}$ & $\begin{array}{l}\text { How will the Next } \\
\text { Generation Change the } \\
\text { Business World? A Report } \\
\text { on a Survey. }\end{array}$ & 2011 & EEUU & Empírico & & & & & & $x$ \\
\hline 27 & Wos & $\begin{array}{l}\text { Deal J.J., } \\
\text { Altman D.G., } \\
\text { Rogelberg } \\
\text { S.G. }\end{array}$ & $\begin{array}{l}\text { Millennials at Work: What } \\
\text { We Know and What We } \\
\text { Need to Do (If Anything) }\end{array}$ & 2010 & EEUU & Teórico & & & & & & $x$ \\
\hline 28 & Wos & $\begin{array}{l}\text { Wong, } \\
\text { M., Gardiner, } \\
\text { E., Lang, } \\
\text { W., Coulon, L. }\end{array}$ & $\begin{array}{l}\text { Generational differences in } \\
\text { personality and motivation: } \\
\text { Do they exist and what are } \\
\text { the implications for the } \\
\text { workplace? }\end{array}$ & 2008 & Australia & Empírico & & & & & $x$ & \\
\hline 29 & Wos & $\begin{array}{l}\text { Smola K.W., } \\
\text { Sutton C.D. }\end{array}$ & $\begin{array}{l}\text { Generational differences: } \\
\text { Revisiting generational } \\
\text { work values for the new } \\
\text { millennium }\end{array}$ & 2002 & EEUU & Empírico & $x$ & & & & & \\
\hline 30 & Wos & $\begin{array}{l}\text { Cennamo L., } \\
\text { Gardner D. }\end{array}$ & $\begin{array}{l}\text { Generational differences in } \\
\text { work values, outcomes and } \\
\text { person-organisation values fit }\end{array}$ & 2008 & $\begin{array}{l}\text { Nueva } \\
\text { Zelanda }\end{array}$ & Empírico & & & & $x$ & & \\
\hline 31 & Scopus & $\begin{array}{l}\text { Rasila H., } \\
\text { Rothe P. }\end{array}$ & $\begin{array}{l}\text { A problem is a problem is } \\
\text { a benefit? Generation Y } \\
\text { perceptions of open-plan } \\
\text { offices }\end{array}$ & 2012 & Finlandia & Empírico & & & & & & $x$ \\
\hline 32 & Scopus & $\begin{array}{l}\text { Kong H., } \\
\text { Wang S., Fu X. }\end{array}$ & $\begin{array}{l}\text { Meeting career expectation: } \\
\text { can it enhance job satisfac- } \\
\text { tion of Generation Y? }\end{array}$ & 2015 & China & Empírico & & $x$ & $x$ & $x$ & $x$ & $x$ \\
\hline 33 & Scopus & $\begin{array}{l}\text { Hernaus T., } \\
\text { Vokic N.P. }\end{array}$ & $\begin{array}{l}\text { Work design for different } \\
\text { generational cohorts: } \\
\text { Determining common and } \\
\text { idiosyncratic job character- } \\
\text { istics }\end{array}$ & 2014 & Croacia & Empírico & & $x$ & $x$ & $x$ & $x$ & \\
\hline 34 & Scopus & Galagan P. & Engaging generation & 2006 & Inglaterra & Empírico & & & $x$ & $x$ & $x$ & $x$ \\
\hline 35 & Scopus & Meola C.C. & $\begin{array}{l}\text { Addressing the needs of } \\
\text { the Millennial workforce } \\
\text { through equine assisted } \\
\text { learning }\end{array}$ & 2016 & EEUU & Teórico & & & & & $x$ & $x$ \\
\hline
\end{tabular}




\begin{tabular}{|c|c|c|c|c|c|c|c|c|c|c|c|c|}
\hline & \\
\hline & & & & & & & \multirow[b]{2}{*}{ 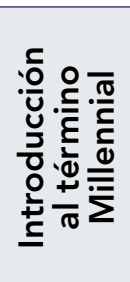 } & \multicolumn{4}{|c|}{ Características } & \multirow[b]{2}{*}{ 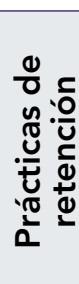 } \\
\hline \# & Base & Autor & Título & Año & $\begin{array}{l}\text { Lugar del } \\
\text { estudio }\end{array}$ & $\begin{array}{l}\text { Tipo de } \\
\text { Estudio }\end{array}$ & & $\begin{array}{l}\frac{n}{0} \\
\frac{0}{0} \\
\frac{0}{0} \\
\frac{u}{n} \\
0\end{array}$ & 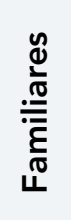 & $\frac{\mathscr{d}}{\frac{\pi}{U}}$ & $\begin{array}{l}\frac{\mathscr{d}}{\pi} \\
\frac{0}{0} \\
\frac{0}{\pi}\end{array}$ & \\
\hline 36 & Scopus & Williams E. & $\begin{array}{l}\text { Driven to Distraction: } \\
\text { How Electronic Media Are } \\
\text { Affecting the Brain and the } \\
\text { Implications for Human } \\
\text { Resource Development in } \\
\text { the Future }\end{array}$ & 2012 & EEUU & Teórico & & & & $x$ & & $x$ \\
\hline 37 & Scopus & $\begin{array}{l}\text { Tews M.J., } \\
\text { Michel J., Xu } \\
\text { S., Drost A.J. }\end{array}$ & $\begin{array}{l}\text { Workplace fun matters ... } \\
\text { but what else? }\end{array}$ & 2015 & EEUU & Empírico & & & $x$ & $x$ & $x$ & $x$ \\
\hline 38 & Scopus & $\begin{array}{l}\text { Schönebeck J., } \\
\text { Schönebeck } \\
\text { M. }\end{array}$ & $\begin{array}{l}\text { Engagement and retention: } \\
\text { Introduction and overview }\end{array}$ & 2016 & Alemania & Teórico & & & & & & $x$ \\
\hline 39 & Scopus & Schaetzle R.J. & $\begin{array}{l}\text { Engagement and retention: } \\
\text { Essentials of rewards and } \\
\text { recognition }\end{array}$ & 2016 & Alemania & Teórico & & & & & & $x$ \\
\hline 40 & Scopus & $\begin{array}{l}\text { [No author na- } \\
\text { me available] }\end{array}$ & $\begin{array}{l}\text { Great expectations: } \\
\text { Recruiting and retaining the } \\
\text { Millennial generation }\end{array}$ & 2016 & Canadá & Empírico & & & $x$ & & $x$ & $x$ \\
\hline 41 & Scopus & Fong H.W.B. & $\begin{array}{l}\text { Factors Influencing } \\
\text { Retention of Gen Y and } \\
\text { Non-Gen Y Teachers } \\
\text { Working at International } \\
\text { Schools in Asia }\end{array}$ & 2018 & EEUU & Empírico & & & $x$ & $x$ & & $x$ \\
\hline 42 & Scopus & $\begin{array}{l}\text { Kumar K., } \\
\text { Velmurugan R. }\end{array}$ & $\begin{array}{l}\text { A study on the work life } \\
\text { balance of Generation Y } \\
\text { Information Technology (IT) } \\
\text { employees in Cochin }\end{array}$ & 2018 & India & Empírico & & & & & & \\
\hline 43 & Scopus & $\begin{array}{l}\text { Glazer S., } \\
\text { Mahoney } \\
\text { A.C., Randall } \\
\text { Y. }\end{array}$ & $\begin{array}{l}\text { Employee development's } \\
\text { role in organizational } \\
\text { commitment: a preliminary } \\
\text { investigation comparing } \\
\text { generation X and millennial } \\
\text { employees }\end{array}$ & 2019 & EEUU & $\begin{array}{l}\text { Teórico y } \\
\text { Empírico }\end{array}$ & $x$ & $x$ & $x$ & $x$ & $x$ & $x$ \\
\hline 44 & Scopus & $\begin{array}{l}\text { Zaharee M., } \\
\text { Lipkie T., } \\
\text { Mehlman S.K., } \\
\text { Neylon S.K. }\end{array}$ & $\begin{array}{l}\text { Recruitment and Retention } \\
\text { of Early-Career Technical } \\
\text { Talent: What Young } \\
\text { Employees Want from } \\
\text { Employers A study of the } \\
\text { workplace attributes that } \\
\text { attract early-career workers } \\
\text { suggests that Millennials } \\
\text { may not be so different from } \\
\text { earlier generations. }\end{array}$ & 2018 & Inglaterra & $\begin{array}{l}\text { Teórico y } \\
\text { Empírico }\end{array}$ & & & & $x$ & $x$ & $x$ \\
\hline
\end{tabular}




\begin{tabular}{|c|c|c|c|c|c|c|c|c|c|c|c|c|}
\hline & \multirow{2}{*}{\multicolumn{5}{|c|}{ Características }} & \multirow[b]{3}{*}{ 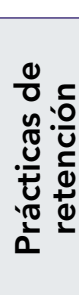 } \\
\hline \multirow[b]{2}{*}{ \# } & \multirow[b]{2}{*}{ Base } & \multirow[b]{2}{*}{ Autor } & \multirow[b]{2}{*}{ Título } & \multirow[b]{2}{*}{ Año } & \multirow[b]{2}{*}{$\begin{array}{l}\text { Lugar del } \\
\text { estudio }\end{array}$} & \multirow[b]{2}{*}{$\begin{array}{l}\text { Tipo de } \\
\text { Estudio }\end{array}$} & & & & & & \\
\hline & & & & & & & 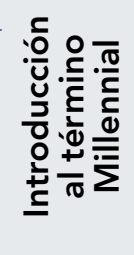 & $\begin{array}{l}\frac{n}{0} \\
\frac{u}{8} \\
\frac{0}{0} \\
\frac{u}{n} \\
\frac{0}{n}\end{array}$ & 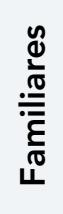 & $\begin{array}{l}\frac{\mathscr{d}}{\pi} \\
\frac{\pi}{U} \\
\dot{0}\end{array}$ & $\begin{array}{l}\frac{\mathscr{d}}{\pi} \\
\frac{0}{0} \\
\frac{0}{0}\end{array}$ & \\
\hline 45 & Scopus & $\begin{array}{l}\text { Madara S.R., } \\
\text { Maheshwari } \\
\text { P., Selvan C.P. }\end{array}$ & $\begin{array}{l}\text { Future of millennial genera- } \\
\text { tions: A review }\end{array}$ & 2018 & $\begin{array}{l}\text { Emiratos } \\
\text { Árabes } \\
\text { Unidos }\end{array}$ & Teórico & & $x$ & & $x$ & $x$ & $x$ \\
\hline 46 & Scopus & $\begin{array}{l}\text { Teng L.S., } \\
\text { Jayasingam } \\
\text { S., Zain } \\
\text { K.N.M. }\end{array}$ & $\begin{array}{l}\text { Debunking the myth of } \\
\text { money as motivator in a } \\
\text { multigenerational workforce }\end{array}$ & 2018 & Malasia & Empírico & $x$ & & & & $x$ & $x$ \\
\hline 47 & Scopus & $\begin{array}{l}\text { García G.A., } \\
\text { Gonzales- } \\
\text { Miranda } \\
\text { D.R., Gallo } \\
\text { O., Roman- } \\
\text { Calderon J.P. }\end{array}$ & $\begin{array}{l}\text { Employee involvement and } \\
\text { job satisfaction: a tale of the } \\
\text { millennial generation }\end{array}$ & 2019 & Colombia & $\begin{array}{l}\text { Teórico y } \\
\text { Emprírico }\end{array}$ & $x$ & & & $x$ & $x$ & $x$ \\
\hline 48 & Scopus & Ohlrich K. & $\begin{array}{l}\text { Exploring the impact of CSR } \\
\text { on talent management with } \\
\text { generation } Y\end{array}$ & 2015 & EEUU & Empírico & & & $x$ & & $x$ & $x$ \\
\hline 49 & Wos & $\begin{array}{l}\text { Mohapatra, } \\
\text { AK; Saxena, } \\
\text { A; Joshi, D; } \\
\text { Chaturvedi, N }\end{array}$ & $\begin{array}{l}\text { Does Job Security Matter } \\
\text { for Generation Y? A } \\
\text { Behavioural Analysis }\end{array}$ & 2017 & India & $\begin{array}{l}\text { Teórico y } \\
\text { Emprírico }\end{array}$ & & $x$ & & & $x$ & $x$ \\
\hline 50 & Scopus & $\begin{array}{l}\text { Anshul A., } \\
\text { Pathak P., } \\
\text { Singh S. }\end{array}$ & $\begin{array}{l}\text { Turnover intention among } \\
\text { generation Y: A study of } \\
\text { coal sector }\end{array}$ & 2018 & India & Empírico & & & & & $x$ & $x$ \\
\hline 51 & Scopus & $\begin{array}{l}\text { Prihadi K.D., } \\
\text { Chang C.K.W., } \\
\text { Lyann G. }\end{array}$ & $\begin{array}{l}\text { Keeping millennials from } \\
\text { quitting due to work stress: } \\
\text { The roles of mattering, } \\
\text { commitment and entrepre- } \\
\text { neurship orientation }\end{array}$ & 2021 & Malasia & Empírico & $x$ & $x$ & & & $x$ & $x$ \\
\hline 52 & Scopus & $\begin{array}{l}\text { Pasko R., } \\
\text { Maellaro R., } \\
\text { Stodnick M. }\end{array}$ & $\begin{array}{l}\text { A study of millennials' } \\
\text { preferred work-related attri- } \\
\text { butes and retention }\end{array}$ & 2021 & EEUU & Empírico & $x$ & & & & $x$ & $x$ \\
\hline 53 & Scopus & $\begin{array}{l}\text { Hassan M.M., } \\
\text { Jambulingam } \\
\text { M., Narayan } \\
\text { E.A.L., Islam } \\
\text { S.N., Zaman } \\
\text { A.U. }\end{array}$ & $\begin{array}{l}\text { Retention Approaches of } \\
\text { Millennial at Private Sector: } \\
\text { Mediating Role of Job } \\
\text { Embeddedness }\end{array}$ & 2021 & Bangladesh & Empírico & & & & & & $x$ \\
\hline 54 & Wos & $\begin{array}{l}\text { Younas, M } \\
\text { and Bari, MW }\end{array}$ & $\begin{array}{l}\text { The relationship between } \\
\text { talent management } \\
\text { practices and retention of } \\
\text { generation 'Y' employees: } \\
\text { mediating role of compe- } \\
\text { tency development }\end{array}$ & 2020 & Pakistán & Empírico & $x$ & & & $x$ & $x$ & $x$ \\
\hline
\end{tabular}




\begin{tabular}{|c|c|c|c|c|c|c|c|c|c|c|c|c|}
\hline \multirow[b]{2}{*}{ \# } & \multirow[b]{2}{*}{ Base } & \multirow[b]{2}{*}{ Autor } & \multirow[b]{2}{*}{ Título } & \multirow[b]{2}{*}{ Año } & \multirow[b]{2}{*}{$\begin{array}{l}\text { Lugar del } \\
\text { estudio }\end{array}$} & \multirow[b]{2}{*}{$\begin{array}{l}\text { Tipo de } \\
\text { Estudio }\end{array}$} & \multirow[b]{2}{*}{ 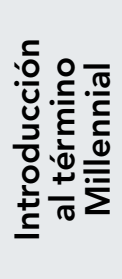 } & \multicolumn{4}{|c|}{ Características } & \multirow[b]{2}{*}{ 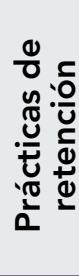 } \\
\hline & & & & & & & & $\begin{array}{l}\frac{n}{0} \\
\frac{u}{0} \\
\frac{0}{0} \\
\frac{u}{n} \\
0\end{array}$ & 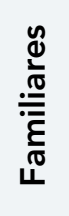 & $\begin{array}{l}\frac{\mathscr{d}}{\pi} \\
\frac{\pi}{U} \\
\dot{0}\end{array}$ & $\begin{array}{l}\frac{\mathscr{d}}{\pi} \\
\frac{0}{0} \\
\frac{0}{0}\end{array}$ & \\
\hline 55 & Wos & $\begin{array}{l}\text { Magni, F and } \\
\text { Manzoni, B }\end{array}$ & $\begin{array}{l}\text { Generational Differences } \\
\text { in Workers' Expectations: } \\
\text { Millennials Want More of } \\
\text { the Same Things }\end{array}$ & 2020 & Italia & Empírico & $x$ & & & $x$ & $x$ & $x$ \\
\hline 56 & Wos & $\begin{array}{l}\text { Hassan, } \\
\text { MDM and } \\
\text { Jambulingam, } \\
\text { M and Alagas, } \\
\text { EN and Uzir, } \\
\text { MUH and Al } \\
\text { Halbusi, H }\end{array}$ & $\begin{array}{l}\text { Necessities and Ways of } \\
\text { Combating Dissatisfactions } \\
\text { at Workplaces Against the } \\
\text { Job-Hopping Generation Y } \\
\text { Employees }\end{array}$ & 2020 & Bangladesh & Empírico & $x$ & & & & $x$ & $x$ \\
\hline
\end{tabular}

Fuente: elaboración propia con base en la revisión de literatura.

\subsection{Caracterización}

Teniendo en cuenta la cantidad de características halladas durante la revisión y con el objetivo de facilitar su entendimiento, fue necesario agruparlas en cuatro grandes grupos: psicológicas, familiares, sociales y comportamiento en el ámbito laboral.

\subsubsection{Características psicológicas}

En términos psicológicos, los millennials parecen tener una urgente sensación de inmediatez y son impacientes por tener éxito (Barkhuizen, 2014; Navós, 2015; Ng et al., 2010; Thompson \& Gregory, 2012). Ante los cambios producidos cuando ingresan al mercado laboral, como lo son las largas horas de trabajo, la poca seguridad laboral ante la reducción de personal, la sobrecarga de trabajo y la ambigüedad de roles, los millennials responden desfavorablemente con aumentos significativos en sus niveles de ansiedad y depresión (Kumar \& Velmurugan, 2018; Madara et al., 2018; Ng et al., 2010; Twenge \& Campbell, 2008). Los más jóvenes de esta generación son menos resistentes y vulnerables al agotamiento, estrés laboral y otras enfermedades de salud mental. Debido a esto, durante la contingencia por el Covid-19, el aislamiento preventivo y la falta de retroalimentación social a la que están acostumbrados, esta característica pudo ser mucho más evidente, afectando sus niveles de rotación (Prihadi et al., 2021). 
Por otra parte, se hace énfasis en que son personas con autoestima exagerada, que tienen grandes expectativas para su desarrollo laboral (promociones y recompensas), poca paciencia y voluntad (Hernaus \& Vokic, 2014; Thompson \& Gregory, 2012) y mucha confianza en sí mismos (Jonck et al., 2017; Mohapatra et al., 2017). Esta nueva generación de trabajadores es considerada individualista (De \& Su, 2011; Rayan, 2011; Twenge, 2010; Twenge \& Campbell, 2008). Sin embargo, autores como Navós (2015) y Muttoni (2011) sostienen que los millennials cuentan con habilidades para trabajar en grupos diversos, como lo menciona este último autor en su artículo "Buscan empresas donde sentirse bien y desarrollarse".

\subsubsection{Características familiares}

Los padres de esta nueva generación son los baby boomers, individuos que optaron por tener menos hijos y contaban con buenos recursos para mantenerlos, razón por la que se dedicaban a criarlos y sobreprotegerlos (Fong, 2018; Glass, 2007). De allí se acuñan dos términos: primero "padres helicóptero", por ser padres que siempre están pendientes de sus hijos y sobrevolando tanto en su vida personal como académica, lo cual les generó a largo plazo dificultades para tomar decisiones profesionales (Cennamo \& Gardner, 2008; Howe \& Strauss, 2007; Navós, 2015; Thompson \& Gregory, 2012; Twenge \& Campbell, 2008). Igualmente, estos padres les proporcionaban retroalimentación positiva y la certeza de nunca estar equivocados. De allí nace el segundo término: "hijos trofeo", ya que en los partidos o actividades culturales, sin importar si los hijos ganaban o perdían, estos siempre recibían un trofeo (Galagan, 2006; Glass, 2007; Navós, 2015; Tews et al., 2015). Estos dos términos se pueden traducir en lo que algunos llamarían optimismo. Sin embargo, hay autores que los consideran como falsa confianza en sí mismos y una necesidad constante de que les respeten sus derechos (Hernaus \& Vokic, 2014; Kumar \& Velmurugan, 2018).

Otra característica en el ámbito familiar es el individualismo e independencia, lo cual se percibe como consecuencia del alto número de divorcios; el temprano ingreso a las guarderías y su permanencia en ellas por gran parte del tiempo; por estar solo la mitad del día acompañados por sus padres (latchkey children); y por el entorno tecnológico en el que se criaron. Así mismo, son individuos que han sido orientados al logro debido a la presión constante de la sociedad y sus padres (Bell \& Mcminn, 2011; De \& Su, 2011; Ng et al., 2010). 
Contrario a lo anterior, algunos autores muestran su preocupación sobre los adultos jóvenes, aquellos que aún mantienen lazos cercanos con sus familias y se demoran en entrar a la fuerza de trabajo, fenómeno que han denominado "adultez emergente", es decir, aquella población que retrasa su entrada a las funciones y responsabilidades de la edad adulta ("Great expectations..." 2016; Hernaus \& Vokic, 2014; Lyons et al., 2012; Shragay \& Tziner, 2011; Twenge \& Campbell, 2008).

Los adultos jóvenes valoran mucho el tiempo libre y la vida personal porque vieron y vivieron muy de cerca el desequilibrio entre el trabajo-vida de sus padres, quienes trabajaban por largas horas (García Lombardía et al., 2011; Kleinhans et al., 2015; Kong et al., 2015). En respuesta a que sus padres hicieron su trabajo pero no les apasionaba, esta generación de trabajadores millennials quiere encontrar pasión por lo que hacen (Ohlrich, 2015).

\subsubsection{Características sociales}

En cuanto a aspectos sociales, los autores coinciden en que estos individuos son nativos de la tecnología y cuentan con mayor educación (Bell \& Mcminn, 2011; Cennamo \& Gardner, 2008; Chacko et al., 2012; De \& Su, 2011; Galagan, 2006; Rayan, 2011; Howe \& Strauss, 2007; Kleinhans et al., 2015; Kong et al., 2015; Teng et al., 2018; Thompson \& Gregory, 2012; Wong et al., 2008), "El manejo de las nuevas tecnologías es inherente a su forma de vida, no solo profesional, también la cotidiana" (García Lombardía et al., 2008, p. 49;). Así mismo, prefieren utilizar fuentes digitales para adquirir conocimiento y en menor medida prefieren la interacción cara a cara (Younas \& Bari, 2020). De igual manera, las redes sociales les permiten establecer relaciones globales, difuminando los límites geográficos para esta generación (Magni \& Manzoni, 2020).

Por otro lado, los millennials buscan equilibrio entre su vida laboral y personal para destinar tiempo a actividades que involucran la responsabilidad social. Estos trabajadores buscan flexibilidad de horarios para poder integrar actividades extra laborales, como estudios, deportes, hobbies o voluntariado en alguna ONG (Hernaus \& Vokic, 2014; Howe \& Strauss, 2007; Navós, 2015; Ng et al., 2010; Pasko et al., 2021). Sin embargo, otros autores no concuerdan y, en cambio, expresan que la ayuda social es importante para los millennials pero no más de lo que es o fue para trabajadores de otras generaciones (Twenge, 2010). 
En cuanto a hechos históricos, esta generación fue testigo a temprana edad de actos terroristas como los vividos el 11 de septiembre de 2001, generando en ellos mentalidad social y un inconmensurable valor por la vida (Glass, 2007; Ng et al., 2010); "son los ciudadanos del mundo en lugar de limitarse a su país de origen" (De \& Su, 2011, p. 2); se consideran multirraciales y multiculturales y por esta razón buscan entornos que les permitan conocer otras culturas, lo cual está a su alcance gracias a internet.

En el proceso de detección de estas características sociales, cabe destacar que los autores Jonck (2017) y Navós (2015) señalan como un limitante, que la muestra de la mayoría de investigaciones está compuesta por personas de clase socioeconómica media, en países desarrollados.

\subsubsection{Características en el ámbito laboral}

En el ámbito laboral, los millennials tienen la necesidad de retroalimentación constante y reconocimiento; buscan tener contacto con los líderes que toman decisiones dentro de la organización y aprender desde su experiencia (Barkhuizen, 2014; García Lombardía et al., 2011; Howe \& Strauss, 2007; Lyons et al., 2012; Navós, 2015; Ng et al., 2010; Pasko et al., 2021; Stanimir, 2015; Thompson \& Gregory, 2012; Twenge \& Campbell, 2008). Esperan un enfoque participativo de gestión, con una comunicación mucho más plana, en la que sus ideas también sean escuchadas y puedan aportar en la toma de decisiones (Pasko et al., 2021). Además, buscan un crecimiento rápido a posiciones altas sin tanto esfuerzo (porque no les gusta trabajar horas extras), para ellos el equilibrio entre su vida laboral y personal es algo primordial (Barkhuizen, 2014; Bissola \& Imperatori, 2010; Carpenter \& De Charon, 2014; Erickson, 2009; Hassan et al., 2020; Kim et al., 2009; Levenson, 2010; Ng et al., 2010; Thompson \& Gregory, 2012).

Esta población es propensa a cambiar constantemente de trabajo, lo cual puede deberse a varias situaciones: primero, estudiaron varios años, se endeudaron y cuando salieron al mercado laboral encontraron pocos trabajos bien remunerados, lo que a la larga afecta su lealtad hacia las organizaciones (Levenson, 2010; Navós, 2015). Segundo, si consideran que sus valores propios chocan o no se relacionan con los valores organizacionales (Ng et al., 2010). Tercero, si no son halagados o no se les brinda reconocimiento, no dudan en buscar otra organización que sí lo haga (Twenge \& Campbell, 2008). Finalmente, tienen 
dificultad para enfrentar la frustración y en ocasiones esto puede ser un detonante para abandonar su cargo (Thompson \& Gregory, 2012).

En un estudio realizado a 1000 trabajadores de una empresa en Italia, se cocluye que los millennials tienen más expectativas que los de otras generaciones. Los autores argumentan que puede ser debido a que aún son nuevos en las organizaciones, tienen confianza y no se han desencantado de ellas. Este resultado lo obtienen luego de controlar la edad, experiencia laboral y permanencia en el trabajo (Magni \& Manzoni, 2020). Entre las expectativas, llama la atención que el salario alto y el pago por desempeño son las dimensiones menos esperadas, mientras que el poder y responsabilidad, el equilibrio trabajo-vida, la retroalimentación, el desarrollo de carrera, la atmósfera social, la capacitación y la seguridad laboral están por encima.

En cuanto a la recompensa, los millennials no solo esperan altos salarios, sino salarios justos; prefieren trabajos que los desafíen y los diviertan a la vez, que les genere un rápido avance dentro de la compañía (Barkhuizen, 2014; Navós, 2015; Ng et al., 2010; Tews et al., 2015). Consideran importante la seguridad laboral (Magni \& Manzoni, 2020; Pasko et al., 2021); buscan un ambiente de trabajo enriquecedor, con prestigio social, en el que puedan brindar algo a la sociedad y buscan aprender cosas nuevas constantemente (Barkhuizen, 2014; Hassan et al., 2020; Wong et al., 2008).

Según la investigación realizada por Kowske, Rasch y Wiley (2010), en la que comparan a las generaciones, se determinó que los millennials tienen niveles más altos de satisfacción y desarrollo profesional que la media, en comparación con las generaciones anteriores (Barkhuizen, 2014; Carpenter \& De Charon, 2014; Kuyken, 2012; Zaharee et al., 2018) y tienen un concepto del éxito basado en la calidad y nivel de su trabajo, sus relaciones o conexiones y la organización de la cual forman parte (Pasko et al., 2021).

La generación del milenio, gracias al desarrollo tecnológico, se puede catalogar como la población con más posibilidades de acceso a la educación, por lo que a esta generación multitareas se le facilita más los procesos de innovación (Bell \& Mcminn, 2011; García Lombardía et al., 2008; Jonck et al., 2017; Navós, 2015; Stanimir, 2015). Son más abiertos al cambio, por esto logran adaptarse fácilmente. Aprenden de manera rápida, fácil, prefieren 
tener un propósito social en cada una de las tareas que realizan, cooperan en equipo y comparten conocimientos (García Lombardía et al., 2011; Kim et al., 2009; Ng et al., 2010).

\subsection{Recomendaciones para la creación de prácticas de retención}

De acuerdo con un estudio de la Society for Human Resource Management-sHrm (2011), el costo de reemplazar y contratar nuevo personal puede ser tan alto como el $60 \%$ del salario anual del empleado; y si se incluye la capacitación y la pérdida de productividad, puede alcanzar un rango de $90 \%$ a $200 \%$ del salario anual de un empleado. Ante estas cifras, es necesario que las empresas sean conscientes de la importancia de entender a la nueva generación de trabajadores, no solo para estar informados, sino para prepararse y estudiar cuidadosamente qué los motiva y qué les impide desarrollar sus funciones adecuadamente dentro de la organización.

Por esta razón, durante la revisión de la literatura, se han mencionado algunas recomendaciones para mejorar la retención de esta generación de trabajadores. A continuación, la tabla 3 sintetiza y relaciona las expectativas asociadas a las características de la generación del milenio con su respectiva recomendación según los autores: 
Tabla 3. Recomendaciones según expectativas asociadas a los Millennials

\section{No. Expectativa Asociada Recomendación para generar estrategias de retención}

Elaboración de planes de reclutamiento especiales, que se enfoquen en los principales motivadores de los trabajadores. Así mismo, la construcción de instructivos o manuales de funciones claros y concisos, con el fin de facilitarle a esta generación, su curva de aprendizaje e incrementar su

*Les gusta rotar de puestos de trabajo para aprender constantemente (Lyons et al., 2012; Thompson \& Gregory, 2012).

* Buscan la oportunidad de ampliar sus horizontes a través de la movilidad laboral y las asigna-

1 ciones internacionales (Levenson, 2010; Ng et al., 2010).

* Esperan un enfoque participativo de gestión, con una comunicación más plana, en la que sus ideas también sean escuchadas y puedan aportar en la toma de decisiones (Pasko et al., 2021). productividad (De \& Su, 2011; Ohlrich, 2015).

Mostrarles el valor e impacto de su trabajo en la organización y hacerlo interesante (Gallicano, 2013; “Great expectations...", 2016; Navós, 2015).

Un enfoque de "comunidades de conocimiento" dentro de un mismo grupo de edad puede ayudar a comprender mejor los contextos reales de las empresas (Kuyken, 2012).

Invertir en el capital humano para generar nuevos conocimientos y habilidades (capacitaciones y tutorías) (Bissola \& Imperatori, 2010; Ohlrich, 2015; Williams, 2012; Younas \& Bari, 2020).

Promover programas como la tutoría inversa, que consiste en que los empleados millennials sean mentores de los adultos mayores en temas como el uso de tecnología y las redes sociales, fomentando así la confianza y el desarrollo de ambos trabajadores (Younas \& Bari, 2020).

Ofrecer una variedad de oportunidades para avanzar en sus carreras, las cuales no siempre estarán ligadas al crecimiento vertical (Navós, 2015).

Capacitar en liderazgo a los gerentes para establecer relación con los millennials y solucionar problemas de manera colaborativa (Pasko et al., 2021). Impulsar el liderazgo transformacional (Hassan et al., 2021).

Crear ambientes de trabajo más relajados, flexibles o divertidos y ofrecer nuevos beneficios: más días de vacaciones, eventos sociales y tiempo para trabajos voluntarios (Barkhuizen, 2014; Navós, 2015; Twenge et al., 2010; Zaharee et al., 2018). Otra propuesta en cuanto al entorno de trabajo consiste en implementar oficinas de planta abierta (Rasila \& Rothe, 2012).

Realizar una combinación de incentivos, con los que ya se cuente (De \& Su, 2011).

Brindar recompensas no solo financieras, por ejemplo: proporcionar relación directa con los gerentes de la compañía, con el fin de que puedan aprender e ir capacitando a futuros líderes (De \& Su, 2011; Kong et al., 2015; Mohapatra et al., 2017; Navós, 2015; Thompson \& Gregory, 2012). Tener un estilo de liderazgo menos jerárquico, más horizontal y menos controlador; aplicar modelos de mentoría (Carpenter \& De Charon, 2014; Fong, 2018; Madara et al., 2018; Muttoni, 2011; Navós, 2015; Pasko et al., 2021). 


\section{No. Expectativa Asociada}

*Buscan equilibrio entre su vida laboral y personal para destinar tiempo a actividades que involucran hobbies y la responsabilidad social (Barkhuizen, 2014; Bissola

3 \& Imperatori, 2010; Carpenter \& De Charon, 2014; Erickson, 2009; Kim et al., 2009; Levenson, 2010a; Ng et al., 2010; Thompson \& Gregory, 2012; Wong et al., 2008; Prihadi et al., 2021).

\section{Recomendación para generar estrategias de retención}

Brindar flexibilidad en cuanto a horarios y metodologías de trabajo, buscar el equilibrio entre la vida personal y laboral para incrementar su creatividad, desarrollar más habilidades y experiencias que los motiven generando lealtad hacia la organización (Barkhuizen, 2014; Carpenter \& De Charon, 2014; Deal et al., 2010; Hassan et al., 2020; Kleinhans et al., 2015; Kumar \& Velmurugan, 2018; Madara et al., 2018; Muttoni, 2011; Navós, 2015; Twenge, 2010).

Fomentar un clima organizacional ético y amigable con la familia (Hassan et al., 2020)

Las empresas deben analizar qué estrategias crear a partir de:

*Considerar su impacto en la sociedad

*Considerar su impacto en el medio ambiente (las empresas deben ser líderes en sostenibilidad ambiental) (Bell \& Mcminn, 2011; "Great expectations..." 2016; Ohlrich, 2015).

Brindar proyectos que se relacionen con sus motivadores y empoderarlos (García et al., 2019; García Lombardía et al., 2011).
Buscar espacios para brindar retroalimentación, ya que es clave para el desarrollo personal y profesional (Glazer et al., 2019; Schaetzle, 2016; Twenge \& Campbell, 2008).

Brindar reconocimiento de sus habilidades en el trabajo (Barkhuizen, 2014; De \& Su, 2011; García Lombardía et al., 2011; Schaetzle, 2016; Schönebeck \& Schönebeck, 2016). Thompson \& Gregory, 2012; Twenge \& Campbell, 2008).

*Son nativos de la tecnología, multitarea, se les facilitan los procesos de innovación y cuen-

6 tan con mayor educación (Bell \& Mcminn, 2011; García Lombardía et al., 2008; Jonck et al., 2017; Navós, 2015; Stanimir, 2015).
Proveerles acceso a la tecnología (Muttoni, 2011).

Los resultados sugieren que estos nuevos empleados, están listos para un salto tecnológico. Es necesario solicitar más sistemas de e-HR "interactivos", capaces de satisfacer las necesidades de los empleados más capacitados, ya que brindar herramientas tecnológicas tiene un impacto significativamente positivo en el trabajador (Bissola \& Imperatori, 2010). Los millennials deben asumir la responsabilidad de recomendar mejoras en los procesos, mediante el aprovechamiento de la tecnología y utilizando una actitud de cuestionamiento (Carpenter \& De Charon, 2014).
*Son personas que tienen grandes expectativas para su desarrollo laboral (promociones y recompensas) (Thompson \& Gregory, 2012).

7 * Tienen niveles más altos de satisfacción y desarrollo profesional que la media, en comparación con las generaciones anteriores (Barkhuizen, 2014; Carpenter \& De Charon, 2014; Kuyken, 2012; Zaharee et al., 2018).
Los gerentes deberían realizar reuniones uno a uno con los millennials para determinar dónde se ven a sí mismos en seis meses, dentro de la organización (Galagan, 2006).

Para la promoción se debe tener en cuenta: ser mentores, estar incluidos en la nueva planificación y tener reuniones sobre objetivos a largo plazo (Carpenter \& De Charon, 2014; Fong, 2018; Plessis et al., 2015).

Tener planes de sucesión dentro de la organización (Barkhuizen, 2014).

Brindar la oportunidad de trabajos interesantes con crecimiento y desarrollo profesional (compromiso de continuidad), llegando a trabajar con grandes personas (compromiso afectivo ) y trabajando en un buen ambiente (Gallicano, 2013; Glazer et al., 2019; Mohapatra et al., 2017; Ohlrich, 2015; Plessis et al., 2015; Zaharee et al., 2018). 


\begin{tabular}{|c|c|c|}
\hline No. & Expectativa Asociada & Recomendación para generar estrategias de retención \\
\hline 8 & $\begin{array}{l}\text { *Se frustran y en ocasiones ese } \\
\text { puede ser un detonante para } \\
\text { salir de una organización (Navós, } \\
\text { 2015; Thompson \& Gregory, } \\
\text { 2012). }\end{array}$ & $\begin{array}{l}\text { Evitar la ambigüedad de rol porque cuando los empleados malinterpre- } \\
\text { tan sus roles, son propensos a agotarse y abandonar la organización. La } \\
\text { ambigüedad del rol puede afectar negativamente el desempeño laboral } \\
\text { de los empleados (Anshul et al., 2018; Kim et al., 2009). }\end{array}$ \\
\hline 9 & $\begin{array}{l}\text { * Disminuir la ansiedad, la } \\
\text { depresión y el estrés (Kumar \& } \\
\text { Velmurugan, 2018; Madara et al., } \\
\text { 2018; Ng et al., 2010; Schönebeck } \\
\text { \& Schönebeck, 2016; Twenge \& } \\
\text { Campbell, 2008; Hassan et al., } \\
\text { 2021). }\end{array}$ & $\begin{array}{l}\text { Implantar políticas y prácticas de RRHH innovadoras que modifiquen las } \\
\text { condiciones ambientales del lugar de trabajo, proporcionar incentivos } \\
\text { para mejorar la salud de la nueva generación de trabajadores y así dismi- } \\
\text { nuir costos y evitar el ausentismo (Anshul et al., 2018; Deal et al., 2010). } \\
\text { Impulsar programas de capacitación sobre inteligencia emocional y ma- } \\
\text { nejo del estrés para mejorar las relaciones entre estos trabajadores y sus } \\
\text { jefes ya que tienen diferentes expectativas (Hassan et al., 2020; Hassan et } \\
\text { al., 2021; Pasko et al., 2021). }\end{array}$ \\
\hline
\end{tabular}

Fuente: elaboración propia a partir de la revisión de literatura.

\section{Discusión}

Los documentos que se analizaron en esta revisión, tal como se presentan en la tabla 1, son en su mayoría estudios realizados con población de clase media, en países desarrollados, en mayor medida de Estados Unidos y solo se identificaron cinco artículos de tres países latinoamericanos. Esto genera una gran cantidad de elementos a considerar incluso para próximas investigaciones, ¿hasta qué punto estas características pueden describir adecuadamente a los millennials en Colombia? considerando que no todos cuentan con los recursos para entrar a formar parte de esta generación. Un ejemplo de esto son aquellos jóvenes que, si bien concuerdan con la edad señalada por los autores, viven en zonas apartadas con grandes brechas de desigualdad, falta de educación y carencia de recursos (entre ellos la tecnología). Aunque las cifras respaldan la alta rotación en los trabajadores millennials, también es necesario considerar qué elementos pueden llevarlos a tomar esa decisión, condiciones laborales, nivel de escolaridad, etc. y contemplar las características propias del mercado laboral y elementos culturales que difieren de los países desarrollados.

Luego de establecer la caracterización de los millennials, es claro que hay una necesidad de entender qué motiva a esta generación para que consideren permanecer en un puesto de trabajo. Son muchas las características que los autores han plasmado en sus artículos, algu- 
nas se han probado empíricamente y otras de forma teórica. Algunas concuerdan y otras se contradicen, por ejemplo: los autores afirman que los millennials son personas flexibles y dispuestas al cambio, pero a su vez tienden a frustrarse fácilmente y esto puede suceder naturalmente ante cualquier cambio (Navós, 2015; Thompson \& Gregory, 2012). También hay quienes afirman que son individualistas e independientes (Bell \& Mcminn, 2011; De \& Su, 2011; Ng et al., 2010), pero en el primer caso, autores como García Lombardía et al. (2011) y Kim et al. (2009) por el contrario resaltan su capacidad de cooperar y trabajar en equipo; y, en el segundo caso, consideran que no son tan independientes y se preocupan por el fenómeno de "adultez emergente" por el que les cuesta asumir responsabilidades y tienen una relación estrecha con los "padres helicóptero", quienes están prestos a ayudarles a tomar decisiones ("Great expectations..." 2016; Hernaus \& Vokic, 2014; Lyons et al., 2012; Shragay \& Tziner, 2011; Twenge \& Campbell, 2008).

Dentro de las recomendaciones para retener a esta generación, expuestas en la tabla 2, es necesario resaltar que en su mayoría se centran en incrementar los procesos de aprendizaje (De \& Su, 2011; García Lombardía et al., 2011; Hassan et al., 2020; Kong et al., 2015; Mohapatra et al., 2017; Navós, 2015; Lyons et al., 2012; Ohlrich, 2015; Thompson \& Gregory, 2012); en el desarrollo de carrera (Gallicano, 2013; Glazer et al., 2019; Mohapatra et al., 2017; Ohlrich, 2015; Plessis et al., 2015; Schaetzle, 2016; Thompson \& Gregory, 2012; Twenge \& Campbell, 2008; Zaharee et al., 2018); y no tanto en prácticas de recompensas o retribución, contrario a lo que podría pensarse en primera instancia (Barkhuizen, 2014; Navós, 2015; Ng et al., 2010; Tews et al., 2015). En cuanto a las recompensas, llama la atención que el salario no se encuentra en el primer lugar de las expectativas de esta generación (Magni \& Manzoni, 2020) y que las recompensas no financieras como el reconocimiento y retroalimentación tienen mayor protagonismo (De \& Su, 2011; Kong et al., 2015; Mohapatra et al., 2017; Navós, 2015; Thompson \& Gregory, 2012).

Dentro de las prácticas de retención también sobresale: primero, el impulso a los trabajadores brindando las herramientas tecnológicas necesarias para esta generación, que ha tenido mayor contacto con los avances en cuanto al uso de internet, uso de redes sociales, manejo de aplicaciones y programas, etc. (Bissola \& Imperatori, 2010; Carpenter \& De Charon, 2014; Muttoni, 2011); segundo, los planes de sucesión, que cobran relevancia más aún para el problema que se ha planteado en el presente artículo (Barkhuizen, 2014), para estar preparados ante el cambio generacional que es inminente; y tercero, están aquellas 
recomendaciones que, si bien se habían realizado años atrás, son clave durante la actual emergencia sanitaria, como es el caso de implantar políticas que motiven el cuidado de la salud de los trabajadores llevando a reducir costos y evitar el ausentismo (Anshul et al., 2018; Deal et al., 2010); brindar alternativas de flexibilidad para lograr equilibrio entre el trabajo y la vida (Barkhuizen, 2014; Carpenter \& De Charon, 2014; Deal et al., 2010; Hassan et al., 2020; Kleinhans et al., 2015; Kumar \& Velmurugan, 2018; Madara et al., 2018; Muttoni, 2011; Navós, 2015; Twenge, 2010); y proponer, organizar y ejecutar programas de capacitación sobre inteligencia emocional y manejo del estrés (Hassan et al., 2020; Hassan et al., 2021; Pasko et al., 2021).

Todas estas recomendaciones son innovadoras, variadas, logran atender las necesidades de los millennials, y no necesariamente requieren de una alta inversión de dinero, sino de tiempo y planeación. Pero cabe aclarar que no son generalizables, no se cuenta con evidencia clara del impacto que podrían generar en la nueva fuerza de trabajo y en los costos que generen o reduzcan. Es en este punto en el que se exhorta a realizar futuras investigaciones.

En cuanto a la rotación es necesario contemplar que, si bien la mayoría de organizaciones suelen optar por retener a los trabajadores, son ellas quienes podrán considerar las estrategias aquí recopiladas y sintetizadas, para incorporarlas en sus prácticas de RRHH. Pero también están aquellas empresas que ven la rotación de trabajadores jóvenes como una oportunidad para obtener perspectivas frescas e innovadoras. Para este tipo de organizaciones también es recomendable conocer las características de esta generación, para así atraer el mejor talento (Magni \& Manzoni, 2020; Pasko et al., 2021).

Dentro de las expectativas a resaltar, las organizaciones deberán prestar especial atención a la capacitación, el desarrollo de los trabajadores y brindar seguridad laboral, ya que hoy en día la forma de contratación tiende a alejarse del tradicional contrato a tiempo completo y a tiempo indefinido (Hassan et al., 2020; Pasko et al., 2021).

Finalmente, teniendo en cuenta lo mencionado por Prihadi et al. (2021), es importante identificar cómo la coyuntura de la pandemia por el Covid-19 ha modificado la conducta de los millennial en temas de rotación. Algunos se vieron en la obligación de postergar su cambio de trabajo para mantenerse estables durante la contingencia y otros, por el contrario, rotaron mucho más ante las condiciones laborales de sobrecarga de trabajo, altos niveles 
de estrés, incertidumbre, agotamiento e inestabilidad en cuanto a salud mental (Prihadi et al., 2021). La falta de equilibrio entre el trabajo y la vida personal también ha sido un detonante para dejar las organizaciones, ante la sobrecarga de tareas del hogar y del trabajo, y la falta de desconexión. Los trabajadores también han optado por rotar debido a la pérdida de lealtad hacia las empresas que tomaron la decisión de despedir una alta cantidad de trabajadores o bien ante la solicitud de las organizaciones de volver a la presencialidad, entre otras cosas.

Si bien no era objetivo de este estudio hacer énfasis en los cambios presentados durante la emergencia sanitaria, se recomienda continuar con la investigación de las características de los millennials, para identificar si han cambiado o si se han ajustado por la coyuntura actual. Sería interesante estudiar, en futuras investigaciones, aquellos casos de jóvenes millennials que ingresaron al mercado laboral por primera vez, pero mediante la virtualidad. En cuanto a las prácticas de retención, podría ser relevante identificar cuáles se están utilizando actualmente, cuáles han cobrado mayor relevancia y cuáles se han dejado de lado, para atender otros problemas propios de la pandemia como la salud de los colaboradores, el afrontamiento, la resiliencia, pérdidas económicas, despidos, entre otros cambios derivados del aislamiento y el trabajo en casa.

\section{Conclusiones e implicaciones gerenciales}

La generación de millennials se puede definir como aquellas personas que nacieron entre 1980 y el año 2000. Entre sus características psicológicas se puede señalar que son impacientes por tener éxito, tienen una alta autoestima, han crecido con orientación al cumplimiento de logros y a tener éxito. Por esto, pueden llegar a frustrarse fácilmente y a aumentar sus niveles de ansiedad y depresión. Algunos autores se contradicen en lo referente a la sociabilidad de esta generación: para algunos, son individualistas, mientras que otros los conciben con habilidades para trabajar en equipo.

En sus familias los padres siempre los sobreprotegían (padres helicóptero) y los premiaban por todo (hijos trofeo). A lo largo de sus vidas, les han brindado retroalimentación, 
independencia y un ambiente con recursos tecnológicos. Sin embargo, la orientación al logro impulsada por los padres les ha generado presión social, familiar y personal.

Esta generación ha tenido mayor acceso a la educación. Según los autores consultados, son nativos de la tecnología, pero para la autora de este documento, podría considerarse más bien que han crecido a la par con ella (ya que cuando nacieron no contaban con tanta tecnología). Consideran que son multirraciales y multiculturales (gracias al internet y las facilidades en la comunicación), quieren tener equilibrio entre su vida y el trabajo, y destinar tiempo para actividades sociales. En el ámbito laboral, desean tener retroalimentación constante, tener contacto con los líderes de las organizaciones, aspiran ascender rápidamente a altos cargos (más aún si se encuentran en cargos de menor rango y tienen potencial de ascenso), cambian constantemente de trabajo, prefieren trabajos en los que adquieran nuevos conocimientos, en los que los desafíen y los diviertan.

El reto actual para los empleadores consiste, entonces, en buscar estrategias óptimas que les permitan retener la fuerza de trabajo, evitar la fuga de conocimiento y, por ende, la pérdida de productividad. Las prácticas de retención que aquí se presentaron señalan que no es necesario realizar grandes inversiones de dinero, lo que sí es importante es mejorar y fortalecer los procesos que tiene a cargo el área de Recursos Humanos. En síntesis, conocer a la nueva generación de trabajadores será clave para entender cómo mejorar procesos, actualizarse aprovechando las habilidades tecnológicas de esta generación, que les permitan adquirir ventajas competitivas, poder sobrevivir y adaptarse al cambio.

Dentro de las limitaciones de los artículos revisados encontramos que, en su mayoría, los estudios son de corte transversal. Por esta razón, se recomienda para futuras investigaciones realizar estudios longitudinales y en diferentes culturas, que permitan evaluar las características de esta generación y así poder identificar la causalidad de las mismas, ampliando el conocimiento existente al respecto. De igual manera, realizar estudios longitudinales para el caso de las prácticas de retención mencionadas, puesto que también es importante evaluar el impacto de las mismas, en las organizaciones y en diferentes sectores. Otro elemento a considerar para futuras investigaciones es evaluar si las características de la generación se deben a la edad y la falta de experiencia o posiblemente a otro tipo de características como las laborales que también pueden influir, tales como el tipo de contrato, nivel jerárquico en la organización, tipo de labor que desempeña, etc. 
Aun teniendo en cuenta que la revisión sistemática de literatura es un proceso riguroso para identificar lo que se ha escrito sobre un tema, es necesario mencionar como limitación de este trabajo que solo se tomaron en cuenta artículos, no se incluyeron libros o conferencias, ni tampoco se realizó una búsqueda completa en fuentes de literatura gris, es decir que la información aquí plasmada se limita a los hallazgos de documentos científicos presentes en revistas indexadas, que se encuentran en las bases de datos mencionadas durante la metodología.

Finalmente, el aporte de la presente revisión de literatura consiste en sintetizar y poner en perspectiva tanto las características de la Generación Y, como las prácticas de retención que recomiendan diferentes autores, permitiéndole a las organizaciones que conozcan el perfil y las necesidades de sus empleados (Younas \& Bari, 2020), para que se centren en aquello que verdaderamente valoran los trabajadores del milenio y las organizaciones no pierdan esfuerzos o recursos por la falta de direccionalidad y obsolescencia de prácticas de gestión humana. Considerar los elementos recabados en este documento puede permitir a los empleadores abrir una ventana de oportunidad, mejorando la satisfacción, motivación, lealtad y posiblemente reduciendo la intención de rotar de los trabajadores, lo que se traduce en un ahorro de costos por pérdida de productividad, contratación y formación de nuevos empleados (Hassan et al., 2021; Kuyken, 2012; Pasko et al., 2021). 


\section{Anexo 1. Rango de años a los que pertenecen los millennials según los diferentes autores de la revisión de literatura}

\begin{tabular}{|c|c|c|}
\hline Autor & Año inicio & Año final \\
\hline Navós, Oscar & 1982 & 1994 \\
\hline Ng, ESW; Schweitzer, L; Lyons, ST & 1980 & 1995 \\
\hline Howe N., Strauss W. & 1982 & 2005 \\
\hline $\begin{array}{l}\text { Pilar García Lombardía, Guido } \\
\text { Stein, José Ramón Pin }\end{array}$ & 1980 & \\
\hline De H., SuX. & 1980 & 2000 \\
\hline Thompson C., Gregory J.B. & 1980 & \\
\hline Jonck P., van der Walt F., Sobayeni N.C. & 1982 & 2000 \\
\hline Stanimir A. & 1980 & 1999 \\
\hline Twenge, JM & 1982 & \\
\hline $\begin{array}{l}\text { Pilar García Lombardía, Guido } \\
\text { Stein, José Ramón Pin }\end{array}$ & 1982 & 1992 \\
\hline Federico Muttoni & 1980 & 2000 \\
\hline Glass A. & 1976 & 1992 \\
\hline Plessis L.D., Barkhuizen N., Stanz K., Schutte N. & 1980 & \\
\hline Bissola R., Imperatori B. & 1982 & 2003 \\
\hline Lyons S.T., Schweitzer L., Ng E.S.W., Kuron L.K.J. & 1980 & \\
\hline Kim H., Knight D.K., Crutsinger C. & 1977 & 1994 \\
\hline Kuyken K. & 1978 & 1994 \\
\hline Carpenter M.J., de Charon L.C. & 1980 & \\
\hline Levenson A.R. & 1980 & \\
\hline Kleinhans K.A., Chakradhar K., Muller S., Waddill P. & 1982 & 1999 \\
\hline Gallicano T.D. & 1982 & 2002 \\
\hline Bell, Nancy Sutton; Connell, J. A.; McMinn, Nathan E. & 1982 & 2000 \\
\hline Deal J.J., Altman D.G., Rogelberg S.G. & & 2000 \\
\hline Wong, M., Gardiner, E., Lang, W., Coulon, L. & 1982 & 2000 \\
\hline Smola K.W., Sutton C.D. & 1979 & 1995 \\
\hline Cennamo L., Gardner D. & 1980 & 2000 \\
\hline Meola C.C. & 1982 & 2025 \\
\hline Williams E. & 1982 & 2000 \\
\hline S.A. & 1980 & 2000 \\
\hline Fong H.W.B. & 1977 & \\
\hline Kumar K., Velmurugan R. & 1980 & 2000 \\
\hline Glazer S., Mahoney A.C., Randall Y. & 1980 & 1995 \\
\hline Madara S.R., Maheshwari P., Selvan C.P. & 1981 & 1995 \\
\hline
\end{tabular}




\begin{tabular}{lll}
\hline Teng L.S., Jayasingam S., Zain K.N.M. & 1981 & 2001 \\
\hline García G.A., Gonzales-Miranda D.R., Gallo O., Roman-Calderon J.P. & 1980 & 1999 \\
\hline Ohlrich K. & 1980 & 1994 \\
\hline Anshul A., Pathak P., Singh S. & 1981 & 1999 \\
\hline Pasko R., Maellaro R., Stodnick M. & 1980 & 2000 \\
\hline Hassan, MDM and Jambulingam, M and Alagas, EN and Uzir, MUH and Al Halbusi, H & 1981 & 2000 \\
\hline Magni, F and Manzoni, B & 1981 & 1996 \\
\hline Hassan M.M., Jambulingam M., Narayan E.A.L., Islam S.N., Zaman A.U. & 1981 & 2000 \\
\hline Younas, M and Bari, MW & 1981 & 2000 \\
\hline Prihadi K.D., Chang C.K.W., Lyann G. & 1983 & 2000 \\
\hline
\end{tabular}

Fuente: elaboración propia a partir de la revisión de literatura (A partir de este anexo se obtiene el promedio del rango de años a los que pertenecen los millennials para este artículo).

\section{Referencias}

Anshul, A., Pathak, P., \& Singh, S. (2018). Turnover intention among generation Y: A study of coal sector, 2000, 163-170.

Arshed, N., \& Danson, M. (2015). The Literature Review. En R. MacIntosh, \& K. D. O'Gorman (Eds.), Research Methods for Business and Management: A Guide to Writing Your Dissertation (pp. 31-49). Goodfellow Publishers.

Barkhuizen, N. (2014). Exploring the importance of rewards as a talent management tool for Generation Y employees. Mediterranean Journal of Social Sciences, 5(27). https://doi. org/10.5901/mjss.2014.v5n27p1100

Bell, N. S., \& Mcminn, N. E. (2011). How will the Next Generation Change the Business World? A Report on a Survey. Insights Journal, 45.

Bissola, R., \& Imperatori, B. (2010). Generation Y at work: The role of e-HRM in building positive work attitudes. CEUR Workshop Proceedings, 570, 378-399. http://ceur-ws.org/ Vol-570/paper022.pdf

Carpenter, M. J., \& De Charon, L. C. (2014). Mitigating multigenerational conflict and attracting, motivating, and retaining millennial employees by changing the organizational culture: A theoretical model. Journal of Psychological Issues in Organizational Culture, 5(3), 68-84. https://doi.org/10.1002/jpoc.21154

Cennamo, L., \& Gardner, D. (2008). Generational differences in work values, outcomes and person-organisation values fit.Journal of Managerial Psychology, 23(8), 891-906. https:// doi.org/10.1108/02683940810904385 
Chacko, H. E., Williams, K., \& Schaffer, J. (2012). A Conceptual Framework for Attracting Generation $\mathrm{Y}$ to the Hotel Industry Using a Seamless Hotel Organizational Structure. Journal of Human Resources in Hospitality and Tourism, 11(2), 106-122. https://doi.org /10.1080/15332845.2012.648843

Chicaíza-Becerra, L., Riaño-Casallas, M. I., Rojas-Berrio, S. P., \& Garzón-Santos, C. (2017, junio). Revisión Sistemática de la Literatura en Administración. Documentos FCE-CID, Escuela de Administrción y Contadría Pública, (29), 1-18. https://doi.org/10.13140/ RG.2.2.15345.89443

DANE. (2017). Gran Encuesta Integrada de Hogares (GEIH) [Boletín Técnico]. https://www. medellin.gov.co/irj/go/km/docs/pccdesign/medellin/Temas/PlaneacionMunicipal/ Publicaciones/2017/Shared\%20Content/Documentos/GEIH/Informe\%20Anual\%20de\%20 resultados\%202017.pdf

DANE. (2019). Entrega de resultados Censo nacional de población y vivienda (CNPV)- 2018 [Comunicado de Prensa, Censo nacional de población y vivienda (CNPv)]. https://www. dane.gov.co/files/censo2018/informacion-tecnica/cnpv-2018-comunicado-3ra-entrega.pdf

De, H., \& Su, X. (2011, 12-14 de agosto). Managing generation Y: Recruiting and motivating. International Conference on Management and Service Science, MASS 2011. Whan, China. https://doi.org/10.1109/ICMSS.2011.5998114

Deal, J. J., Altman, D. G., \& Rogelberg, S. G. (2010). Millennials at work: What we know and what we need to do (if anything). Journal of Business and Psychology, (25), 191-199. https://doi.org/10.1007/s10869-010-9177-2

Erickson, T. J. (2009). Gen Y in the Workforce. Harvard Business Review, 87(2). https://hbr. org/2009/02/gen-y-in-the-workforce-2

Fong, H. W. B. (2018). Factors Influencing Retention of Gen Y and Non-Gen y Teachers Working at International Schools in Asia. Educational Forum, 82(1), 59-67. https://doi.org/10.10 80/00131725.2018.1379578

Galagan, P. 2006. Engaging generation Y: An interview with Marcus Buckingham. Training E Development, 60(8), 27-30.

Gallicano, T. D. (2013). Relationship management with the Millennial generation of public relations agency employees. Public Relations Review, 39(3), 222-225. https://doi.or$\mathrm{g} / 10.1016 / \mathrm{j}$. pubrev.2013.03.001

García Lombardía, P., Stein, G., \& Pin, J. R. (2008). Motivaciones y valores de la Generación Y. Revista de Antiguos Alumnos del IEEM, 14(3), 47.

García Lombardía, P., Stein, G., \& Pin, J. R. (2011). ¿A qué generación pertenece usted? Revista de Antiguos Alumnos del IEEM, 14(3), 62-66. 
García, G., Gonzales, D. R., Gallo, O., \& Roman, J. P. (2019). Employee involvement and job satisfaction: a tale of the millennial generation. Employee Relations, 41(3), 374-388. https:// doi.org/10.1108/ER-04-2018-0100

Glass, A. (2007). Understanding generational differences for competitive success. Industrial and Commercial Training, 39(2), 98-103. https://doi.org/10.1108/00197850710732424

Glazer, S., Mahoney, A. C., \& Randall, Y. (2019). Employee development's role in organizational commitment: a preliminary investigation comparing generation $\mathrm{X}$ and millenial employees. Industrial and Commercial Training, 51(1), 1-12. https://doi.org/10.1108/ ICT-07-2018-0061

Great expectations: Recruiting and retaining the Millennial generation. (2016). Human Resource Management International Digest, 24(2), 32-35. https://doi.org/10.1108/ HRMID-12-2015-0184

Hassan, M. M., Jambulingam, M., Alagas, E. N., Uzir, M. U. H., \& Halbusi, H. A. (2020). Necessities and ways of combating dissatisfactions at workplaces against the Job-Hopping Generation y Employees. Global Business Review, 1-26. https://doi.org/10.1177/0972150920926966

Hassan, M. M., Jambulingam, M., Narayan, E. A., Islam, S. N., \& Zaman, A. U. (2021). Retention Approaches of Millennial at Private Sector: Mediating Role of Job Embeddedness. Global Business Review. https://doi.org/10.1177/0972150920932288

Hernaus, T., \& Vokic, N. P. (2014). Work design for different generational cohorts: Determining common and idiosyncratic job characteristics. Journal of Organizational Change Management, 27(4), 615-641. https://doi.org/10.1108/JOCM-05-2014-0104

Howe, N., \& Strauss, W. (2007). Los próximos 20 años. Cómo evolucionarán las actitudes de los clientes y de la fuerza de trabajo. Harvard Business Review, 85(7), 40-54. https://dialnet.unirioja.es/servlet/articulo?codigo $=2328570$

Jonck, P., Van der Walt, F., \& Sobayeni, N. C. (2017). A generational perspective on work values in a South African sample. SA Journal of Industrial Psychology, 43(1). https://doi. org/10.4102/sajip.v43.1393

Kim, H., Knight, D. K., \& Crutsinger, C. (2009). Generation Y employees' retail work experience: The mediating effect of job characteristics. Journal of Business Research, 62(5), 548-556. https://doi.org/10.1016/j.jbusres.2008.06.014

Kleinhans, K. A., Chakradhar, K., Muller, S., \& Waddill, P. (2015). Multigenerational perceptions of the academic work environment in higher education in the United States. Higher Education. https://doi.org/10.1007/s10734-014-9825-y 
Kong, H., Wang, S., \& Fu, X. (2015). Meeting career expectation: Can it enhance job satisfaction of generation Y? International Journal of Contemporary Hospitality Management, 27(1), 147-168. https://doi.org/10.1108/IJCHM-08-2013-0353

Kowske, B. J., Rasch, R., \& Wiley, J. (2010). Millennials' (lack of) attitude problem: An empirical examination of generational effects on work attitudes. Journal of Business and Psychology, (25) 265-279. https://doi.org/10.1007/s10869-010-9171-8

Kumar, K., \& Velmurugan, R. (2018). A Study on the Work Life Balance of Generation Y Information Technology (IT) Employees in Cochin. International Journal of Engineering \& Technology, 7(3.6), 142-147. https://doi.org/10.14419/ijet.v7i3.6.14958

Kuyken, K. (2012). Knowledge communities: Towards a re-thinking of intergenerational knowledge transfer. VINE, 42(3-4), 365-381. https://doi.org/10.1108/03055721211267495

Levenson, A. R. (2010). Millennials and the world of work: An economist's perspective. Journal of Business and Psychology, (25), 257-264. https://doi.org/10.1007/s10869-010-9170-9

Lyons, S. T., Schweitzer, L., Ng, E. S. W., \& Kuron, L. K. J. (2012). Comparing apples to apples: A qualitative investigation of career mobility patterns across four generations. Career Development International, 17(4). https://doi.org/10.1108/13620431211255824

Madara, S. R., Maheshwari, P., \& Selvan, C. P. (2018). Future of millennial generations: A review. 2018 Advances in Science and Engineering Technology International Conferences, ASET 2018, 1-4. https://doi.org/10.1109/ICASET.2018.8376927

Magni, F., \& Manzoni, B. (2020). Generational Differences in Workers' Expectations: Millennials Want More of the Same Things. European Management Review, 17(4), 901-914. https:// doi.org/10.1111/emre.12405

Mannheim, K., \& Sanchez, I. (1993, abril-junio). El problema de las generaciones. Reis, (62), 193-242. https://doi.org/10.2307/40183643

Mannheim, K. (1952). The problem of Generations. En P. Kecskemeti (Ed.), Essays on the sociology of knowledge (pp. 276-322). Routledge \& Kegan Paul Ltd. https://marcuse.faculty. history.ucsb.edu/classes/201/articles/27MannheimGenerations.pdf

Mohapatra, A. K., Saxena, A., Joshi, D., \& Chaturvedi, N. (2017). Does Job Security Matter for Generation Y ? A Behavioural Analysis. Pacific Business Review International, 10(5), 92-98. http://www.pbr.co.in/2017/2017_month/Nov/9.pdf

Muttoni, F. (2011). Buscan empresas donde sentirse bien y desarrollarse. Revista de Antiguos Alumnos del IEEM, 14(3), 36.

Navós, O. (2015). Nuevas generaciones en las empresas: algunas claves para su gestión. Horizaontes Empresariales, 14(2), 47-54. http://revistas.ubiobio.cl/index.php/HHEE/ article/view/2632 
Ng, E. S. W., Schweitzer, L., \& Lyons, S. T. (2010). New Generation, Great Expectations: A Field Study of the MillennialGeneration. Journal of Business and Psychology, 25, 281-292. https://doi.org/10.1007/s10869-010-9159-4

Ohlrich, K. (2015). Exploring the impact of CSR on talent management with generation Y. South Asian Journal of Business and Management Cases, 4(1), 111-121. https://doi. org/10.1177/2277977915574044

Pasko, R., Maellaro, R., \& Stodnick, M. (2021). A study of millennials' preferred work-related attributes and retention. Employee Relations, 43(3), 774-787. https://doi.org/10.1108/ER05-2020-0224

Plessis, L. D., Barkhuizen, N., Stanz, K., \& Schutte, N. (2015). The management side of talent: Causal implications for the retention of generation y employees. Journal of Applied Business Research, 31(5), 1767-1780. https://doi.org/10.19030/jabr.v31i5.9390

Prihadi, K. D., Chang, C. K. W., \& Lyann, G. (2021). Keeping millennials from quitting due to work stress: The roles of mattering, commitment and entrepreneurship orientation. International Journal of Public Health Science, 10(2), 445-450. https://doi.org/10.11591/ ijphs.v10i2.20839

Rasila, H., \& Rothe, P. (2012). A problem is a problem is a benefit? Generation Y perceptions of open-plan offices. Property Management, 30(4), 362-375. https://doi. org/10.1108/02637471211249506

Rayan, L. (2011). Generación Y en el día a día. Revista de Antiguos Alumnos del IEEM, 14(3), $50-51$.

Ridley, D. (2012). The Literature Review: A Step-by-Step Guide for Students (2a ed.) [sage Study Skills Series]. Edición Kindle.

Schaetzle, R. J. (2016). Engagement and retention: Essentials of rewards and recognition. En M. Zeuch (Ed.), Handbook of Human Resources Management. Springe. https://doi. org/10.1007/978-3-642-40933-2

Schönebeck, J., \& Schönebeck, M. (2016). Engagement and Retention: Introduction and Overview. En M. Zeuch (Ed.), Handbook of Human Resources Management. Springe. https://doi.org/10.1007/978-3-642-40933-2_51-1

Shragay, D., \& Tziner, A. (2011). The generational effect on the relationship between job involvement, work satisfaction, and organizational citizenship behavior. Revista de Psicologia del Trabajo y de las Organizaciones, 27(2), 143-157. https://doi.org/10.5093/tr2011v27n2a6

Smola, K. W., \& Sutton, C. D. (2002). Generational differences: Revisiting generational work values for the new millennium. Journal of Organizational Behavior, 23(4), 363-382. https://doi.org/10.1002/job.147 
Society for Human Resource Management. (2011). SHRM customized buman capital benchmarking report. SHRM.

Stanimir, A. (2015). Generation Y - characteristics of attitudes on labour market. Mediterranean Journal of Social Sciences, 6(2S5). https://doi.org/10.5901/mjss.2015.v6n2s5p22

Strauss, W., \& Howe, N. (1991). Generations. Harper Perennial.

Teng, L. S., Jayasingam, S., Naim, K., \& Zain, M. (2018). Debunking the Myth of Money as Motivator in a Multigenerational Workforce. Pertanika Journal Social Sciences E Humanities, 26(1), 129-148. http://www.pertanika.upm.edu.my/resources/files/ Pertanika\%20PAPERS/JSSH\%20Vol.\%2026\%20(1)\%20Mar.\%202018/08\%20JSSH-1707-20163rdProof.pdf

Tews, M. J., Michel, J., Xu, S., \& Drost, A. J. (2015). Workplace fun matters ... but what else? Employee Relations, 37(2), 248-267. https://doi.org/10.1108/ER-10-2013-0152

Thompson, C., \& Gregory, J. B. (2012). Managing Millennials: A Framework for Improving Attraction, Motivation, and Retention. The Psychologist-Manager Journal, 15(4). https:// doi.org/10.1080/10887156.2012.730444

Twenge, J. M. (2010). A Review of the Empirical Evidence on Generational Differences in WorkAttitudes. Journal of Business and Psychology, 25, 201-210. https://doi.org/10.1007/ s10869-010-9165-6

Twenge, J. M., \& Campbell, S. M. (2008). Generational differences in psychological traits and their impact on theworkplace. Journal of Managerial Psychology, 23(8), 862-877. https:// doi.org/10.1108/02683940810904367

Twenge, J. M., Campbell, S. M., Hoffman, B. J., \& Lance, C. E. (2010). Generational Differences in Work Values: Leisure and Extrinsic ValuesIncreasing, Social and Intrinsic Values Decreasing. Journal of Management, 36(5), 1117-1142. https://doi.org/10.1177/0149206309352246

Williams, E. (2012). Driven to Distraction: How Electronic Media Are Affecting the Brain and the Implications for Human Resource Development in the Future. Advances in Developing Human Resources, 14(4), 626-639. https://doi.org/10.1177/1523422312455627

Wong, M., Gardiner, E., Lang, W., \& Coulon, L. (2008). Generational differences in personality and motivation: Do they exist and what are the implications for the workplace? Journal of Managerial Psychology, 23(8), 878-890. https://doi.org/10.1108/02683940810904376

Younas, M., \& Bari, M. W. (2020). The relationship between talent management practices and retention of generation ' $\mathrm{Y}$ ' employees: mediating role of competency development. Economic Research-Ekonomska Istrazivanja, 33(1), 1330-1353. https://doi.org/10.1080/ 1331677X.2020.1748510 
Zaharee, M., Lipkie, T., Mehlman, S. K., \& Neylon, S. K. (2018). Recruitment and Retention of Early-Career Technical Talent: What Young Employees Want from Employers A study of the workplace attributes that attract early-career workers suggests that Millennials may not be so different from earlier generations. Research Technology Management, 61(5), 51-61. https://doi.org/10.1080/08956308.2018.1495966 